\title{
New robust observables on Rayleigh waves affected by an underground cavity: from numerical to experimental modelling
}

\author{
C. Filippi, ${ }^{1,2}$ D. Leparoux, ${ }^{1}$ G. Grandjean, ${ }^{2}$ A. Bitri ${ }^{2}$ and Ph. Côte ${ }^{1}$ \\ ${ }^{1}$ French Institute of Science Technology for Transport development and Networks (IFSTTAR), Nantes, France. E-mail: celine.filippi@ifsttar.fr \\ ${ }^{2}$ French Geological Survey (BRGM), Orléans, France
}

Accepted 2019 June 2. Received 2019 May 26; in original form 2018 February 19

\begin{abstract}
SUMMAR Y
The investigation and monitoring of shallow hazards due to the presence of underground cavities remain a challenge for geophysical approaches. Thus, seismic surface waves have been tested in several recent research projects in order to detect and localize voids as well as to determine their geometries. Among these works, numerous numerical studies have proved the feasibility of Rayleigh waves to detect cavities. However, most imagery processes adapted to $R$ waves are faced with difficulties when applying them to real data. This limitation points to a major problem: the interactions between Rayleigh waves and a cavity are complex, particularly in the case of dispersing and attenuating surrounding media. Here, a combined approach based on numerical and experimental data obtained in a reduced-scale measurement bench is conducted to better understand the seismic wave propagation phenomena involved in the presence of a cavity and define robust observables that can be used in field measurements. The observables bearing the cavity signature are studied qualitatively and quantitatively on numerical and experimental recordings. The latter take into account all the propagation phenomena involved. The observations are carried out on the vertical and horizontal component of the Rayleigh wave displacement. The selected observables are studied depending on nondimensional cavity's parameters versus the frequency, that is the wavelength-to-size ratio and the wavelength-to-depth ratio. The effects of the cavity's parameters on the observables show particularities as a function of these components, such as a higher rate of the amplitude on the horizontal component as well as a perturbation of the direct seismic surface wave amplitude above the cavity, also higher on the horizontal component. This latter feature is particularly visible on the variation of the elliptical particle motion recorded at the surface. It can be linked to the mode conversions that occur in the vicinity of the cavity and which predominate on the horizontal component when the signal is normalized.
\end{abstract}

Key words: Numerical modelling; Surface waves and free oscillations; Wave propagation; Wave scattering and diffraction.

\section{INTRODUCTION}

Detecting and positioning underground cavities (karstic cavities, caves, tunnels, etc.) is still challenging for geotechnical projects due to the geological complexity of the subsurface. Therefore the geological hazards of collapsing soil due to shallow cavities down to $20 \mathrm{~m}$ depth are a recurrent issue for land planning. Thus, numerous geophysical research projects over recent decades have focused on developing non-destructive methods for detecting and determining the geometry of shallow cavities.

The wide range of available geophysical methods such as GroundPenetrating Radar (GPR), microgravimetry, resistivity imaging and their combinations (Cardarelli et al. 2002; Mochales et al. 2007;
Riddle et al. 2010; Fehdi et al. 2014), can provide good approximations of underground cavity positioning. However the feasibility of each method is limited by the physical characteristics of the subsurface that depend on its specific geological and anthropogenic contexts. For example, GPR is not efficient in the case of very heterogeneous media, such as backfills, or in that of the high soil conductivity of moist clays and saturated soils. Regarding microgravimetric methods, a large number of applied surveys have demonstrated their capacity to detect shallow cavities (Butler 1984; Bishop et al. 1997). However, the density contrast is too weak if the void diameter is relatively small compared to its depth, making cavity detection difficult. 
In order to overcome these limitations, seismic methods can provide an alternative in geophysical campaigns to locate and characterize shallow underground cavities. The seismic methods tested up to now for voids detection are based on the effects of different kinds of waves on the strong and local contrast between the surrounding medium and the air-filled cavity. Shallow seismic methods applied to cavity detection described in literature are summarized below. Concerning the refraction method based on arrival-time measurements different data sets have shown the presence of voids near the surface (Belfer et al. 1998; Maraio et al. 2015) but in most cases, it fails since the perturbation of the traveltimes obtained is too small to be exploited. This may be due to the fact that the cavity is small compared to the wavelength or because the soil stratification has a bigger influence on traveltime compared to cavity perturbation. The principle of seismic reflection can be effective for imaging deep cavities but for shallow depths, that is the subsurface geotechnical zone, the events reflected are often masked by surface waves (Miller et al. 1991; Fiore et al. 2013; Sloan et al. 2015). This last point makes the extraction of cavity-related information from raw data difficult. Thus, seismic surface waves usually used to investigate near surface zones (Shtivelman et al. 2003; Socco et al. 2004; Foti et al. 2011) could significantly improve the feasibility of seismic methods for detecting underground cavities. However, the state of art concerning research works which involve Rayleigh waves ( $\mathrm{R}$ wave) for cavity detection shows lacks of efficiency as well as remaining uncertainties in the proposed methods. As summarized below, it is due to the complexity of the phenomena and their effects in the presence of a void, which remain not precisely described in the literature.

Indeed, several imaging techniques using the vertical component (V-component) of $R$ waves have been developed to locate cavities in the last decades. Through these different methods, the cavity is characterized in the resulting seismic profile by anomalies in the time and frequency domains caused by back and forward scattering from the cavity. For example, the MASW method Multi-Analysis of Surface Waves, developed from the SASW Spectral Analysis of Surface Waves, can be used to establish variations in seismic shear wave velocity with depth in the presence of a void (Satoh et al. 1991; Gucunski et al. 1996; Luke et al. 1997; Xu et al. 2006). The results showed fluctuations in the dispersion curve caused by wave reflections from the boundaries of the cavity. They affect certain frequencies contained in the spectral band and the Rayleigh wavefield seems to be filtered by the cavity. Based on these observations, Leparoux et al. (2000) and Park et al. (2009) proposed a processing protocol to improve the estimate of the scattered field by stacking data in Common Receiver/Shot Gather configurations following a Dynamic Linear Move Out (DLMO) correction. The lateral cavity position is easily detected from the apex of the scattering hyperbola visible on the seismic signal in time domain. The process has been tested on field data and on a reduced scale test site (Grandjean \& Leparoux 2004) to show the robustness of the method and its capacity to determine the lateral position of the cavity when the depth is low enough to be reached by surface waves. Around the location of the void, the resulting stacked traces showed a zone of lower amplitude caused by destructive interferences. These anomalies in the frequency domain are linked to the depth information by using the fundamental mode as it has been shown by Socco et al. (2015), who determined a linear relationship between frequencies and depth ranges. However, the depth determination of the cavity may remain uncertain if the perturbations alter higher modes in the phase velocity diagram. In addition, Nasseri-Moghaddam et al. (2007) used the position and size of these anomalies due to trapped energy inside the void to link them to location and embedment of the void.
Nonetheless, Putnam et al. (2008) showed the uncertainty on the position of energy concentration areas, leading to ambiguities on the void boundaries locations. Indeed, the anomalies are revealed in seismic recordings for a range of offsets determined empirically in specific contexts. In summary, all these methods revealed anomalies due to a shallow cavity on recordings of the vertical component along direct surface waves which contain the incident part and the forward scattering. Yet, information contained in these anomalies remain difficult to be used as the particular phenomena involved when surface waves reach the cavity are not well known.

Others methods process the diffraction effects of the cavity in time domain. It is noteworthy that, since the effects of the amplitude anomalies seem due to diffraction interferences, it is crucial to analyse the scattering wavefield in order to determine whether a method is capable of detecting and characterizing a void. That is why several numerical and analytical studies were conducted to formulate solutions of the inverse scattering problem. Some authors dealt with the Born approximation for surface waves imaging, which requires the background velocity model and a weak scattering (Snieder 1987; Blonk et al. 1994; Ernst et al. 2002). However, this approximation cannot be done when non-linear effects in the near-field are considered or with strong scattering. In parallel, different methods of migration are adapted. The natural migration which uses recorded data to approximate Green functions for migration was developed and tested with ambient noise and controlled-source by Liu et al. (2017). Hyslop \& Stewart (2015) calculated the frequency dependent reflection coefficient at the position of the lateral heterogeneity by separating the direct $R$ wave and the backscattering. Herman et al. (2000) and Campman et al. (2005) used an approach based on an integral equation formulation to image an object just beneath the line of receivers and took into consideration multiple scattered fields. However, most of these methods don't provide accurate information about the depth or the diameter of the cavity. Indeed, they determine a pseudo-depth calculated from the mapping of frequency to depth of one-third or one-half of a wavelength. Xia et al. (2007) used also a simple equation based on traveltime data from diffraction curve to improve the determination of the top of a void. But, all the complex phenomena highlighted in several numerical studies (e.g. Gelis et al. 2005; Xia et al. 2007; Chai et al. 2012; Dravinski et al. 2013; Shao et al. 2016)) are not taken into account for the seismic imaging. The $R$ wave propagation is simplified and mode conversions are not part of the process. Moreover, the numerical approach applied to homogeneous media systematically shows strong backscattered arrivals due to the cavity. Yet, the diffractions tend to be degraded during the stacking or migration process as it has been pointed by Berkovitch et al. (2009) and Bitri et al. (2016). It may be due to combined effects: the diffraction amplitudes are weak compared to the incident wave front, the intrinsic attenuation is strong in subsurface materials and the dynamic moveout corrections are not able to accurately approximate diffraction traveltime. These limits reveal that the seismic observable based on the surface waves backscattering arrivals is not robust enough to image a cavity in some contexts.

To overcome these problems Gelis (2005), Romdhane et al. (2011), Perez et al. (2012) and Bretaudeau et al. (2012) modelled all the phenomena in an elastic medium containing a cavity and proposed using full-waveform inversion (FWI) as a quantitative inversion process to recover the cavity in the subsurface medium. One variant is the use of the reverse-time-migration (RTM) developped by Almuhaidib et al. (2015) in the presence of voids. They proposed different strategies that have provided good results for numerical data. But the approach remains difficult, with high 
Table 1. Scale ratio between experimental and field data.

\begin{tabular}{lcc}
\hline Parameters & Field data & Experimental data \\
\hline Seismic waves velocity & $\mathrm{V}$ & $2 \mathrm{~V}$ \\
Time & $\mathrm{T}$ & $10^{-3} \mathrm{~T}$ \\
Frequency & $\mathrm{F}$ & $2 \times 10^{3} \mathrm{~F}$ \\
Wavelength & $\mathrm{L}$ & $10^{-3} \mathrm{~L}$ \\
Density & $\mathrm{D}$ & $\mathrm{D}$ \\
Quality factor & $\mathrm{Q}$ & $\mathrm{Q}$ \\
\hline
\end{tabular}

computational cost in the case of field measurements due to the complexity of the subsurface zone. In addition, it is vital to assess the initial model, the issue of local minima makes the process very sensitive, the solution is not unique (Foti et al. 2009) and the choice of parameters has a strong influence on the signal. All these works show the complexity of the phenomena involved which result in the difficulty to develop a robust and efficient imaging method for cavity detection based on surface waves arrivals.

On the basis of these elements, another strategy could be to define a new, robust and simplified imagery method in comparison to FWI, but which also takes into consideration all the complex phenomena involved during wave propagation. To do this, a first stage of study is necessary to determine $R$ wave observables that can be used to both detect and characterize a cavity. This first step is the main goal of the approach proposed here. Consequently, the study presented in this paper aims to define new robust observables from seismic surface measurements that can be used in field campaigns to characterize shallow underground cavities. For that, we propose to analyse the effects of a cavity not only on the V-component but also on the horizontal component (H-component) of the seismic recordings. The horizontal component is taken in the direction of propagation of the wave, that is perpendiculary to the cavity axis. This approach is novel as all the studies cited above only take into consideration the vertical seismic displacements.

A second innovative aspect of the study concerns the experimental approach conducted in a small scale laboratory. Indeed, the measurements are carried out in an ultrasonic laboratory bench called MUSC (Measurement at Ultrasonic SCale). The reduced-scale tool is designed to record all the physical phenomena involved in a controlled environment. It allows us to draw some observables on real seismic data, which bear the cavity signature on the $R$ wave in terms of amplitudes of the scattering part as well as the ellipticity of the particle motion. These results can be identified in a well controlled environment but in real experimental configuration.

Then, a third novelty of our results is provided by a quantitative analysis of the resulting effects depending on the cavity's diameter and depth compared to the propagated wavelength. This nondimensional analysis is provided by numerical simulations in an homogeneous medium. The synthetic seismograms are computed using the open source code EFISPEC3D based on the spectral element method (De Martin 2010). The obtained features on both components depending on the cavity characteristics (size and depth) are described as new observables, which could further help to identify the presence of a void through imaging methods. All these elements involving new analysis of seismic data in the presence of a cavity are successively presented as following: the numerical and experimental tools are described in the first part. Then, experimental data obtained on a 2-layer model are compared to numerical results and qualitative features of the seismic signature on each component are detailed. In the following part, the diffraction features are extracted on numerical simulations to analyse them depending firstly on the component and secondly on the cavity size. And finally, the effects of the cavity on the direct $R$ wave including the forward scattering are identified depending on the cavity depth.

\section{SIMULATION METHODS: NUMERICAL AND EXPERIMENTAL TOOLS}

In the first stage of the study presented, we describe the tools used to generate seismic wavefield in a medium containing a cavity numerically and experimentally. The reduced scale experiments are carried out on a bi-layered model and the experimental recordings are then compared to numerical simulations using the same model in order to cross-validate the two modeling approaches.

\subsection{The reduced-scale measurement bench MUSC}

We carried out the experimental acquisition on the "MUSC" measurement bench (Mesure Ultrasonore sans Contact in French) at reduced-scale. This facility enables using a perfectly known medium which contains a void with a controlled geometry. Kaslilar (2007) also studied the surface response of a heterogeneity in an aluminium block with ultrasonic measurements at reduced scale and demonstrated the feasibility of the method. The specificities of the measurement conditions such as coupling effects are also controlled whereas this is not the case during field acquisitions. Moreover, this measurement bench does not entail high costs in terms of resources and time or the approximations used for numerical 3-D wave propagation. For example, although the numerical model is viscoelastic, the intrinsic attenuation is constant with the frequency range, which may be not the case with the experimental model or with the field data. Therefore, MUSC is a relevant intermediate step between the approximations induced in numerical simulations and the complexity of field measurements. Its technical specificities were described by Bretaudeau et al. (2012). Valensi et al. (2015) has recently validated an upgrade of the recording device: the measurement of the $\mathrm{H}$-component is now available and synchronous with the vertical particule displacement measurement. The general scheme of the bench can be summarized as follows:

(1) A reduced scale model made of epoxy resin simulates the underground geological medium.

(2) Ultrasonic waves are generated by a punctual piezoelectric transducer. The source pulse is controlled by a form generator.

(3) The particule displacement at the surface of the resin model, in the order of a nanometer, is recorded by a laser interferometer. The latter is designed for the frequency range [10 kHz; $20 \mathrm{MHz}$ ] and avoids coupling effects between the receivers and the medium.

(4) The source and receiver positions are defined by two independent arms moved automatically above the medium.

To determine the experimental measurement dimensions, it is necessary to evaluate scale transformations between the numerical and experimental models to maintain the ratio constant between the signal wavelength and the model dimensions. The relationships between laboratory dimensions and real dimensions based on the resin properties are presented in Table 1. The scale ratio for the wavelength is 1000 implying that a distance of $10 \mathrm{~m}$ in reality is $10 \mathrm{~mm}$ in the laboratory. Note that compared to the ratio presented in Pageot et al. (2017), we take into consideration a scale ratio equal to 2 for the seismic velocities. This allows us to simulate near surface media which have low seismic velocities by epoxy resins which have higher seismic velocity due to their stiffness. In this way, the scale 
ratio for the frequencies is 2000 . A central frequency of $100 \mathrm{kHz}$ in the laboratory corresponds to a central frequency of $50 \mathrm{~Hz}$ on the field. The quality factor $Q$ and the density $\rho$ of the medium are the same whichever the considered scale. The mechanical properties of the resin used to model the subsurface provide attenuation characteristics similar to that which are in the materials of the subsurface. With all these scale factors, the seismic acquisitions carried out in MUSC allow us to study the real physical phenomena involved in the wave propagation but in a controlled environment. Note that the piezoelectric transducer response, including coupling effects can slightly modify the source waveform. These phenomena have been already pointed out by Bretaudeau et al. (2011) and further studied by Pageot et al. (2017) who demonstrated their reproducibility. They can be taken into account in numerical simulation by inverting the source wavelet as explained below.

In the next paragraph, we present the model used to perform the numerical simulations used to generate the synthetic data that will be compared to the experimental data.

\subsection{The spectral element method for numerical simulations}

The spectral element method (SEM) is used to generate 3-D synthetic seismograms with the open source code EFISPEC3D developed by De Martin (available on http://www.efispec.free.fr/ website). The SEM has the advantage of obtaining exponential convergence with low numerical dispersion. This method can easily incorporate free-surface topography and simulates the propagation of surface waves accurately in the viscoelastic approximation. To achieve this, the model volume is subdivided into a number of non-overlapping hexahedral elements generated by the Trelis software (www.csimsoft.com/trelis). The shape of the elements is welladapted to the surface topography and to the presence of anisotropy. The wavefield on the elements is then discretized by using highdegree Lagrange polynomials, and the integration on each element is based on the Gauss-Lobatto-Legendre (GLL) integration rule (Komatitsch et al. 1999). The sampling of the volume and the GLL integration result in a diagonal mass matrix in the differential equation that governs the time dependence in the system. This important property significantly reduces complexity and computation cost. To avoid parasite reflections, the code uses the paraxial condition in which absorbance is maximal when the angle of incidence of the wave is equal to $90^{\circ}$ close to the boundary. This absorbing condition is implemented on all the sides of the model except for the free surface. The cavity is simulated by applying a free surface boundary condition already applied to the upper limit of the medium. The mesh is refined in the neighbourhood of the heterogeneity. Moreover, the geometrical parameters of the model are determined using several conditions:

(1) The model is large enough not to take into account the weak parasite reflections from the boundaries.

(2) The size of the elements, $s$, is determined to be sufficiently large to obtain reasonable calculation time and sufficiently small to avoid numerical dispersion and capture small wavelengths in the model. To determine the small wavelength propagated in the medium, we need to take the maximal frequency $f_{\max }$. If we consider a Ricker wavelet with a central frequency of $f_{0}$, the maximal frequency is defined like the frequency for which the spectrum amplitude is inferior to 5 per cent of its maximal value. Consequently we have:

$f_{\max } \simeq 3 * f_{0}$.

So to capture the small wavelength in the medium, the size of mesh needs to be equal to:

$s=\frac{V_{\min }}{3 f_{0}}$,

where $V_{\min }$ is the minimum velocity wave propagating in the layer (which corresponds here to the velocity of $R$ waves) and $f_{0}$ is the central frequency of the source.

(3) The time step must be small enough to capture all the frequencies with good accuracy and provide stable integration in the time domain. It is equal to $10^{-5} \mathrm{~s}$.

An example of the numerical mesh around the cavity is presented in Fig. 1.

For the expected cross-validation between the previous experiments and numerical simulations, we built a meshed 3-D model similar to that made with resins and used in the MUSC experiment. We used non-meshed elements with free-surface boundary conditions rather than a low velocity domain to integrate the cavity in the model. Choosing to model a cavity with the free-surface condition can cause difficulties for the imaging process but does not require specific precautions for the direct problem. To perform the comparison, the effective wavelet source is assessed from the experimental acquisition by using the linear source wavelet estimation method in the frequency domain (Pratt et al. 1999). It is introduced in the numerical simulation to compare the seismic shots. Fig. 2 presents several traces obtained for the V-component in order to compare the numerical and experimental recordings. They are very similar with a correlation coefficient higher than 0.93, although slight differences can be seen due to the quality factors that could be not perfectly determined in the resins. Indeed, $Q$ may not be constant on the frequency band chosen. Nonetheless, the correlation coefficients prove that the traces are well superimposed in terms of amplitude and phase before and above the void located between the offsets at 82 and $87.5 \mathrm{~mm}$, and after it. These similar results indicate a positive cross-validation of the small-scale experimental approach and the numerical one. Consequently, the void is correctly simulated in the numerical simulations by the free-boundaries principle. The model used for the cross-validation and the parameter acquisitions are presented in the next part. It should be noted that, for these kind of dimensions ranges, the cavity can be modelled as a void (i.e. without air inside). Consequently, there is no resonance effect of the cavity as described in the paper of Watkins et al. (1967) and recently discussed by Korneev (2009) and Schneider et al. (2017).

\section{QUALITATIVE ASSESSMENTS OF SEISMIC OBSERVABLES AFFECTED BY AN UNDERGROUND CAVITY IN A TABULAR MEDIUM}

The reduced-scale experiments are carried out on a dispersive and bi-layered model to highlight robust observables of $R$ waves affected by a cavity. In this study, the 2-layer model called Bicperc, is made of two resins, F50-pure resin: $V_{s}=1000 \mathrm{~m} \cdot \mathrm{s}^{-1}, V_{p}=$ $2200 \mathrm{~m} . \mathrm{s}^{-1}, \rho=1300 \mathrm{~kg} . \mathrm{cm}^{-3}$ and LAB1000 resin, respectively: $V_{s}=1400 \mathrm{~m} \cdot \mathrm{s}^{-1}, \quad V_{p}=3000 \mathrm{~m} \cdot \mathrm{s}^{-1}, \rho=1500 \mathrm{~kg} \cdot \mathrm{cm}^{-3}$. Fig. 3 shows the models parameters and the acquisition conditions. It contains a cylindrical void in the second layer $6 \mathrm{~mm}$ deep and has a diameter of $5 \mathrm{~mm}$. Taking into account the scale ratios proposed in 


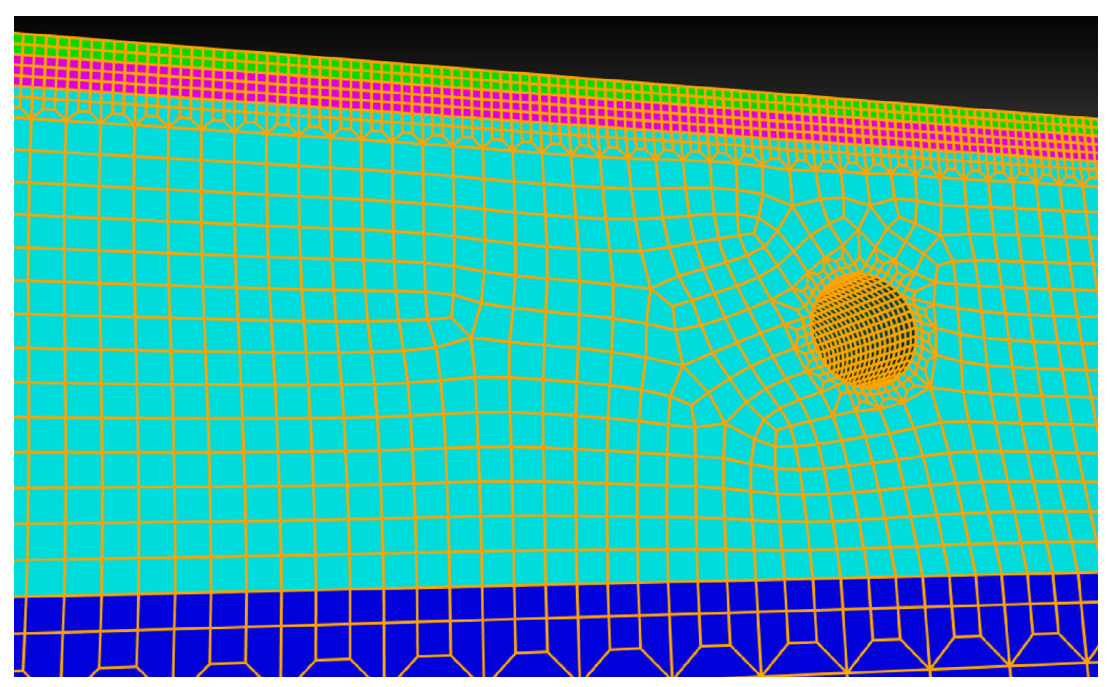

Figure 1. Example of the numerical mesh around the cylindrical cavity. The velocities of the layers represented by the different colours increase with the depth. Consequently, the mesh size is smaller for the superficial layers. The mesh is also refined around the cavity to account for the cavity geometry.

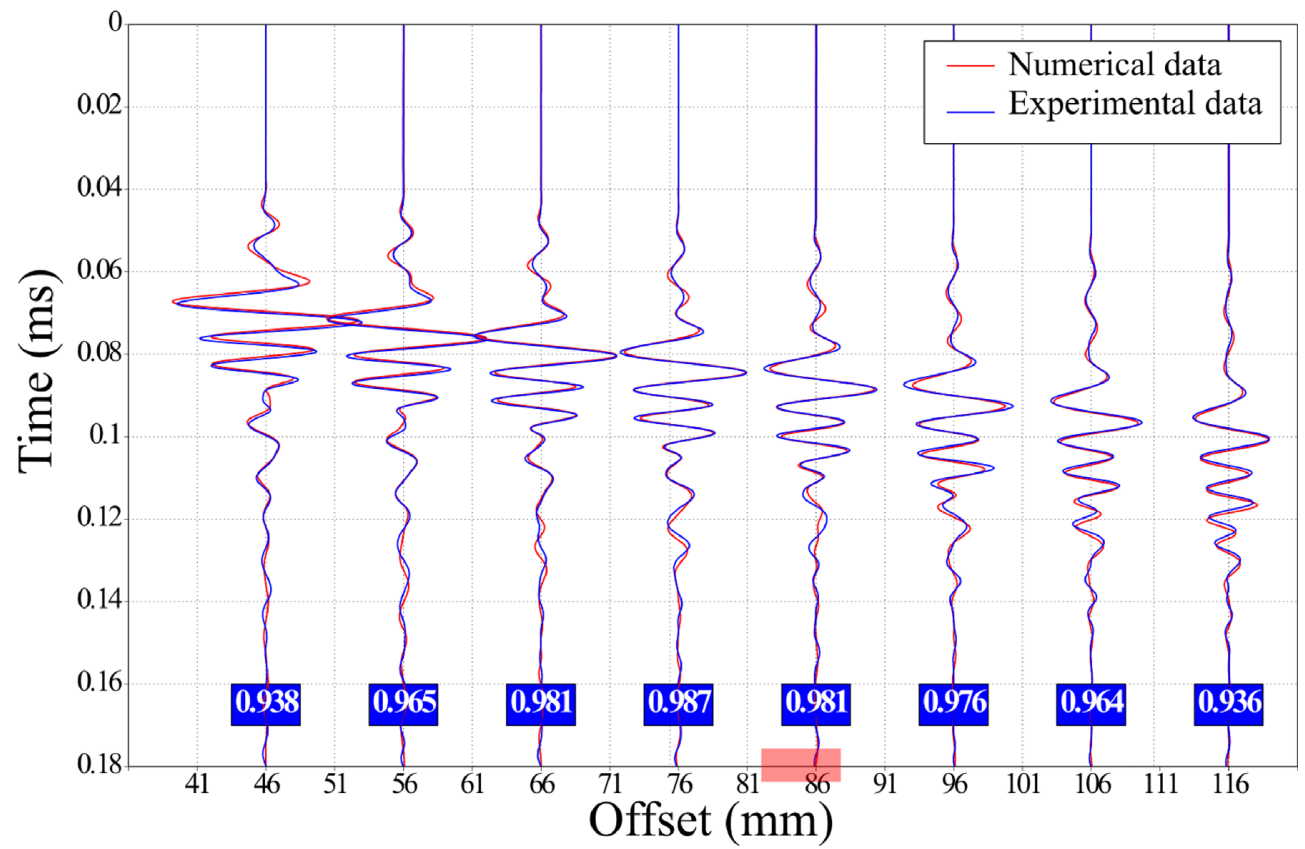

Figure 2. Numerical traces (red) and experimental traces (blue) at different offsets for the V-component. The value in the blue rectangle corresponds to the correlation coefficient between the numerical and experimental traces. The red rectangle corresponds to the projection of the void boundaries at the surface.

Table 1, this model simulates a clay layer of $3 \mathrm{~m}$ thick on limestone including a cavity with a diameter of $5 \mathrm{~m}$ and a $6 \mathrm{~m}$ deep roof (i.e. a realistic context). The attenuation parameters are assessed by the spectral ratio method, assuming that the quality factor is constant in the frequency band considered. The resulting values are: $Q_{s}=$ 15 and $Q_{p}=30$ in the deeper layer (LAB1000 resin) and $Q_{s}=12$ and $Q_{p}=20$ in the upper layer (F50-pure resin). The comparison of the signals shown in Fig. 2 in the previous part, proves that the assumption is valid for the experimental tests used in this study. The frequency and the amplitude of the source wavelet are controlled by selecting an appropriate electrical signal sent to the piezoelectric transducer. The wavelet here is a Ricker wavelet centred on $100 \mathrm{kHz}$, that is $50 \mathrm{~Hz}$ for the simulated field scale. The acquisition set-up is composed of 159 receivers spread across a linear profile $79 \mathrm{~mm}$ long and perpendicular to the cavity axis. The receivers are spaced at $0.5 \mathrm{~mm}$. The distance from the source to the first receiver (minimal offset) equals $L 1=44 \mathrm{~mm}$ and the centre of the cavity axis is located at $L 2=88.5 \mathrm{~mm}$ from the source position (Fig. 3).

Figs 4(a) and (b) show the V and H-component measurements, respectively, carried out in MUSC with prior acquisition in the Bicperc model. The red rectangle corresponds to the cavity's projection at the surface. The signal to noise ratio is much higher for the V-component than for the H-component due to the measurement principle. However, several events and characteristics can be distinguished in both cases as follows:

(1) The arrival recordings of the direct $P$ waves (arrow 1) are clearly distinct from the direct $R$ waves (arrow 2) because the minimal offset $L 1$ between the source and the first receiver is large enough. 


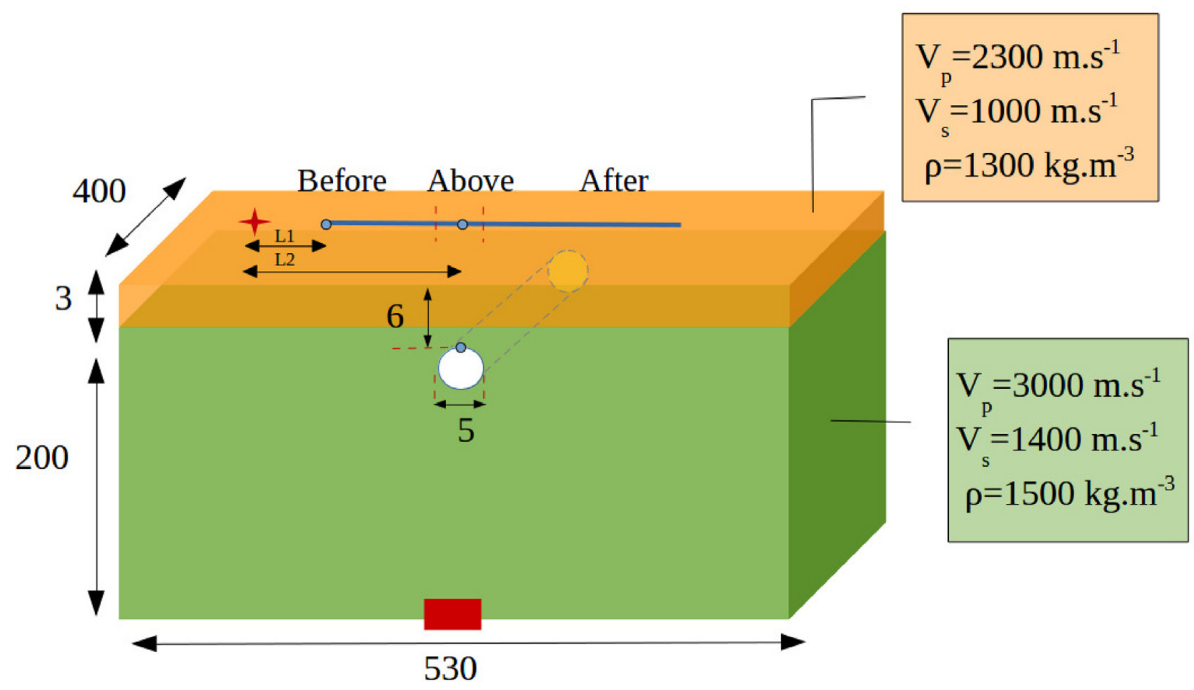

Figure 3. Representation of the experimental Bicperc model. All the dimensions are in millimetres. First layer: $V_{p}=2300 \mathrm{~m} \cdot \mathrm{s}^{-1}, V_{s}=1000 \mathrm{~m} \cdot \mathrm{s}^{-1}, \rho=$ $1300 \mathrm{~kg} \cdot \mathrm{m}^{-3}$. Second layer: $V_{p}=3000 \mathrm{~m} \cdot \mathrm{s}^{-1}, V_{s}=1400 \mathrm{~m} \cdot \mathrm{s}^{-1}, \rho=1500 \mathrm{~kg} \cdot \mathrm{m}^{-3}$.

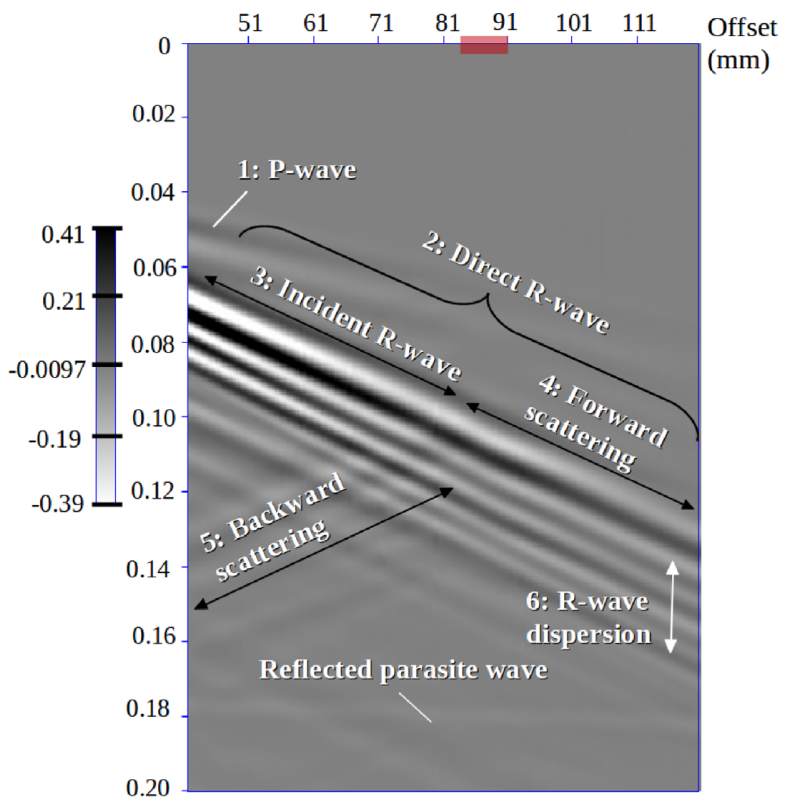

Time (ms)

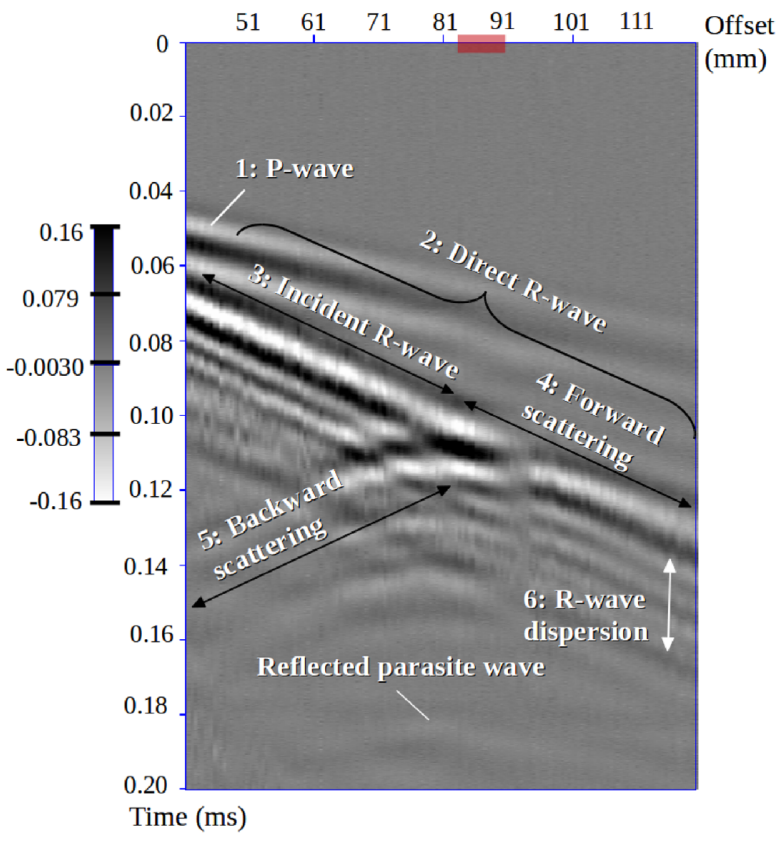

(b)

Figure 4. Seismic recordings obtained in the experimental 2-layer Bicperc model (perc=99.7): (a) V-component ; (b) H-component. The red rectangle corresponds to the projection of the void boundaries at the surface.

(2) The direct $R$ wave (arrow 2) is very visible on each component. Note that it first contains the incident $R$ wave (for receivers before the cavity position) and interferences with the forward scattering wavefield due to the cavity for the last part of the receiver settings. It is difficult to separate the incident wavefield from the forward-scattered one, particularly in the case of real field data in more heterogeneous media. Thus, we will call the direct $R$ wave the combined "incident + forward-scattered" arrivals indicated by arrows 3 and 4 . It will be studied as one observable in the next section.

(3) The direct $R$ wave amplitudes decrease for the receivers just behind the cavity (91-94 mm on the seismic shot), especially for the $\mathrm{H}$-component. However, more distant receivers (after $94 \mathrm{~mm}$ on the seismic shot in Fig. 4b), seem to recover higher amplitudes, even when taking into account the intrinsic and geometrical attenuation.

(4) A slight phase shift occurs on the direct Rayleigh waves, possibly due to the interferences between the incident and the forward scattered part of the Rayleigh wavefield. This phenomenon is particularly noticeable on the V-component.

(5) The backscattering Rayleigh wavefield (arrow 5) is clearly visible on both components but higher on the H-component compared to the direct $R$ wave amplitudes.

(6) The diffraction patterns differ depending on the component. We clearly distinguish the hyperbola shape of the diffraction on the $\mathrm{H}$-component. This feature tends to indicate that mode conversion phenomena occur on the scattering field when the Rayleigh waves 


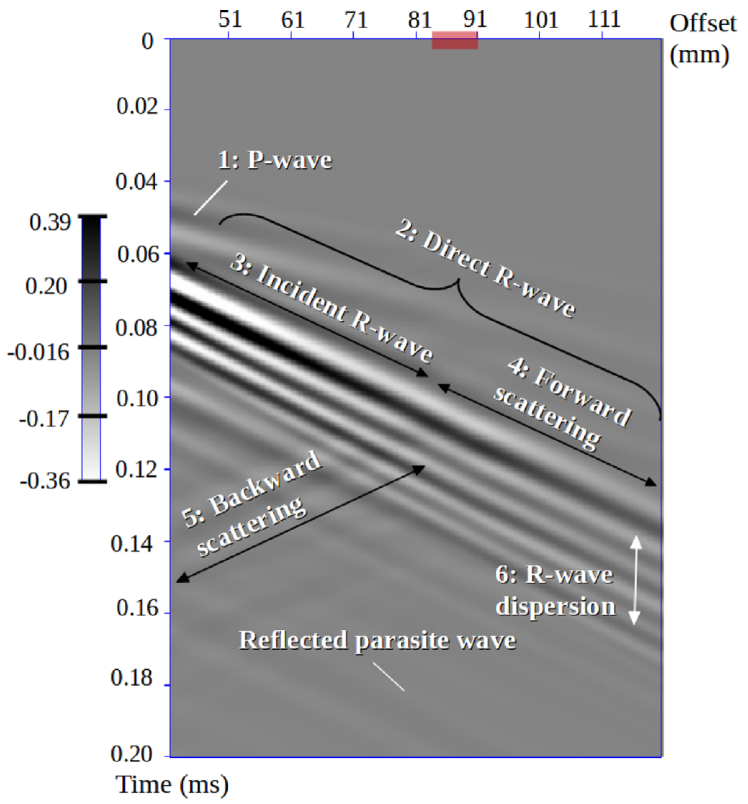

(a)

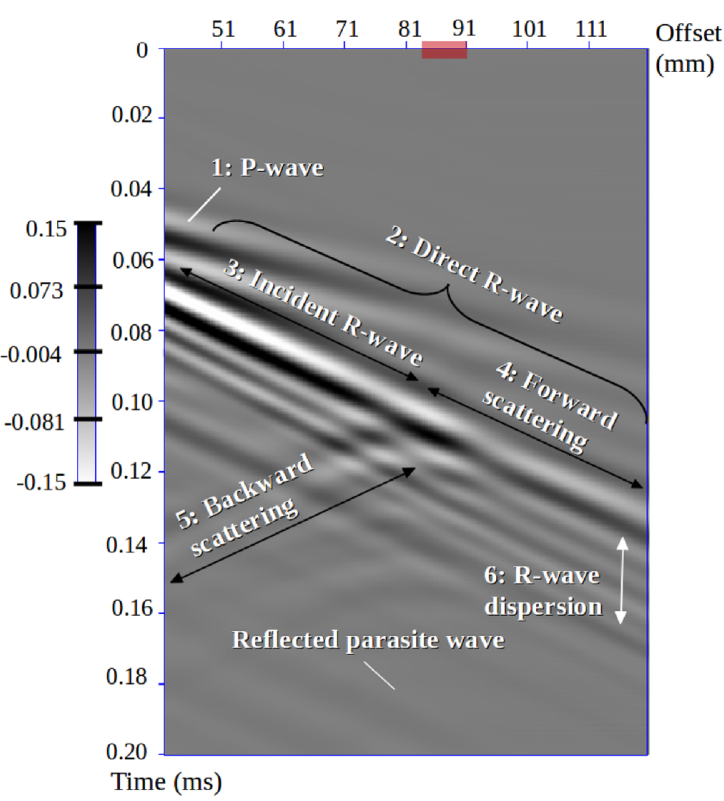

(b)

Figure 5. Seismic recordings obtained in the numerical 2-layer Bicperc model after inserting the effective wavelet obtained with experimental data (perc $=99,7)$ : (a) V-component; (b) H-component. The red rectangle corresponds to the projection of the void boundaries at the surface.

reach the cavity. These phenomena are more dominant for the Hcomponent.

(7) Parasite waves are due to the reflections on the bottom of the model. They correspond to later arrival times. In fact not all the reflections caused by the edges of the resin model disturb the expected seismic recording because the model dimensions are chosen to be large enough.

(8) Ringing effects occur on the direct surface waves arrivals (arrow 6). They are due to the dispersion phenomenon of Rayleigh waves in a context of a 2-layer medium.

Numerical simulations are computed with the same configuration as the previous experimental setup. The seismic recordings obtained with numerical simulations, presented in Fig. 5, confirm the previous experimental observations: the hyperbolic diffraction on the $\mathrm{H}-$ component is clearly visible and its amplitudes are more pronounced compared to the incident $R$ waves than on the V-component. The features highlighted by the small scale experimental model and the numerical simulations confirm the relevance of the H-component since the cavity signature has a singular effect on it. This feature, not described in the literature to our knowledge, is interesting for two reasons: firstly, because we can expect that combining observables carried by $\mathrm{H}$ - and $\mathrm{V}$-components will increase the amount of information and, secondly, because it is not only highlighted in a dispersive medium by numerical simulations but also by experimental ones. This indicates that it could be robust to real measurements in dispersive media. This initial analysis conducted using both experimental and numerical methods, provides credibility for the additional quantitative approach presented in the following part of the paper. Indeed, the cavity parameters [diameter $(s)$, depth $(d)$ ] will be modified in comparison to the seismic wavelength value. The effects on several observables will be studied using numerical tests to facilitate multiplying the model geometries.

\section{ANALYSIS OF DIFFRACTION FEATURES OF $R$ WAVES IN THE PRESENCE OF AN UNDERGROUND CAVITY}

The back-scattered part of Rayleigh waves due to an underground cavity is one of the observables most commonly used to detect a void. It can be used in imaging processes to retro-propagate the wavefield and define the cavitys lateral position (Xia et al. 2007). However, the relation of scattered energy to cavity size is not well known. Moreover, the forward scattering part and the H-component recording are not taken into account, nor are the modes conversions. For all these reasons, we propose here to study the entire scattered wavefield involved when a Rayleigh wave reaches an underground cavity (i.e. in backward and forward directions) on both seismic components. More specifically, our objective is to analyse the diffracted wavefield energy distribution in space and the impact on the components for several cavity dimensions in order to specify the potential information content of the observable. To do this, the numerical simulations are carried out in a homogeneous viscoelastic medium containing a cylindrical cavity. The seismic parameters are defined for the LAB1000 resin used above $\left(V_{s}=\right.$ $\left.1400 \mathrm{~m} . \mathrm{s}^{-1}, V_{p}=3000 \mathrm{~m} . \mathrm{s}^{-1}, \rho=1500 \mathrm{~kg} . \mathrm{cm}^{-3}\right)$. With respect to the dimensions of the model the latter can be considered as semiinfinite (length: $530 \mathrm{~mm} \times$ width : $400 \mathrm{~mm} \times$ depth $: 200 \mathrm{~mm}$ ). As shown in the previous part, reflected events due to the lateral boundaries do not interfere with the seismic arrivals studied and they can be easily windowed in the time domain. Since the medium surrounding the cavity is homogeneous, the study will focus on the interactions between the void and the fundamental mode of the $R$ waves in order to avoid the complexity of the higher modes. The diffraction features previously highlighted in the dispersive Bicperc model are studied only as a function of the cavity and not the surrounding medium. The acquisition parameters are the same as in the previous part (Fig. 3). 


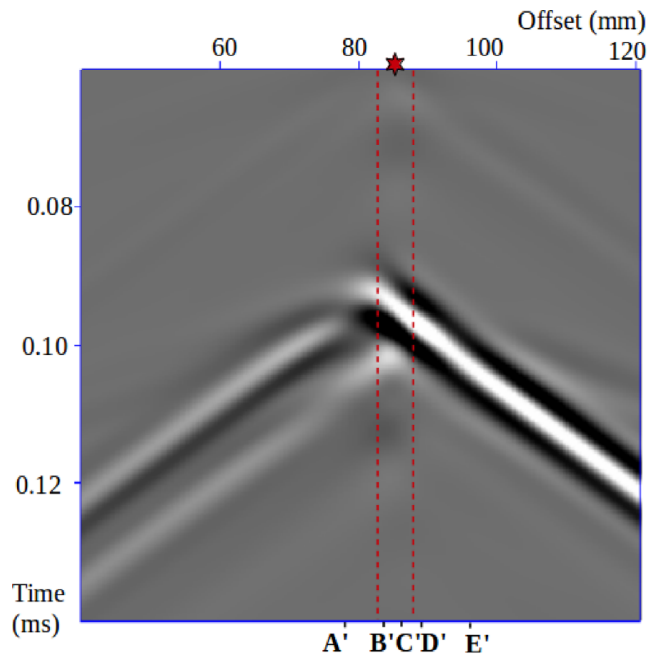

(a)

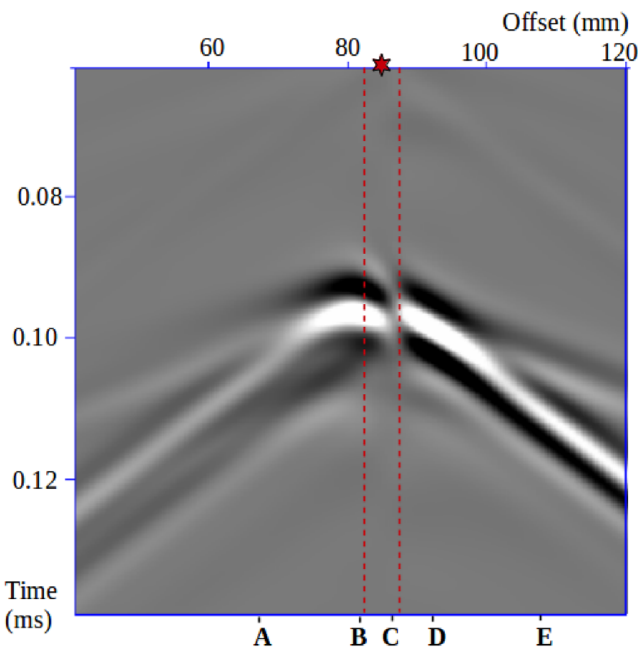

(b)

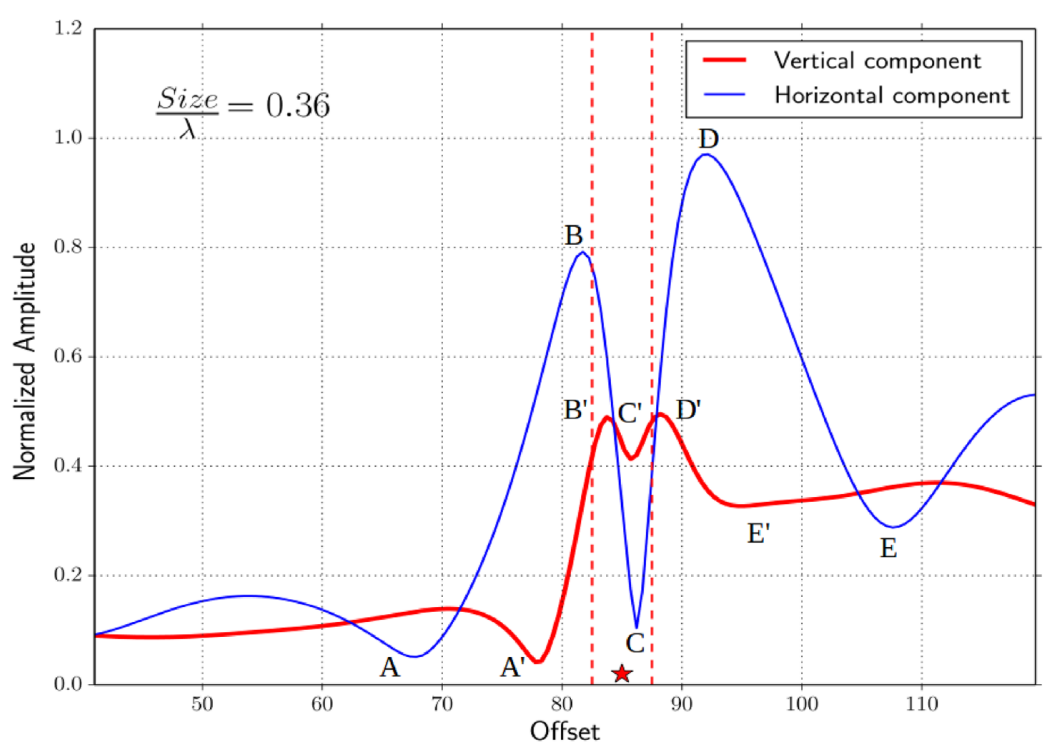

(c)

Figure 6. Amplitudes of the diffracted field for $\frac{\text { Size }}{\lambda}=0.36$ : (a) Diffracted wavefield for the V-component. (b) Diffracted wavefield for the H-component. (c) Curves of the normalized Amplitude for $\frac{\text { Size }}{\lambda}=0.36$. The blue curve corresponds to the H-component and the red one to the V-component. The different letters indicate the amplitude perturbations from $A$ to $E$. The use of a "'” makes reference to the H-component. The red dashed lines define the cavity boundaries.

The total diffracted wavefield is extracted by subtracting the shot gather simulated in a medium containing a cavity from the recording in the same medium but without cavity. The residual data only contain the scattered wavefield pattern due to the cavity. The mesh of the homogeneous model is chosen to be the same as the mesh of the model with a cavity (without considering the elements inside the cavity). Consequently, the residual wavefield is only due to the presence of the cavity and does not depend on the mesh geometry differences between the two surrounding media. Since the diffracted body waves are more strongly attenuated than the $R$ waves for a given propagation distance and since the receivers are located far from the source, the amplitudes of the diffracted P-waves are negligible in comparison to the $R$-wave amplitudes. Thus, we consider the residual scattered wavefield patterns as due only to $R$-wave interactions with the cavity.

We now describe the diffraction features obtained for a propagated seismic frequency equal to $100 \mathrm{kHz}$ in a medium containing a cavity $5 \mathrm{~mm}$ wide and $6 \mathrm{~mm}$ deep. The same geometry as that presented in the previous part in the experimental medium Bicperc is used but this time in an homogeneous medium which is equivalent to a cavity $5 \mathrm{~m}$ wide and a $5 \mathrm{~m}$ deep for central frequency of the source equal to $50 \mathrm{~Hz}$. Thus, Figs 6 (a) and (b) show the resulting residual scattering on both components in this context. The cavity's boundaries are indicated by the red dashed lines. Differences on the diffracted wavefield pattern as a function of the components can be observed. Indeed, the mode conversions ( $R$ waves into body waves) are more visible on the H-component and the shape of the diffraction hyperbola seems more curved on the $\mathrm{H}$ component compared to the V-component. These observations are in accordance with the shot gathers recorded with the measurement bench and presented in the previous part, Fig. 4. Moreover, by looking closely, the diffraction shape is asymmetric: the central axis is shifted towards the lower offset on each component, likely located around the first boundary of the cavity. Another characteristic is that the forward scattering amplitudes are stronger than the amplitudes of the back-scattering for both components, as if the largest 

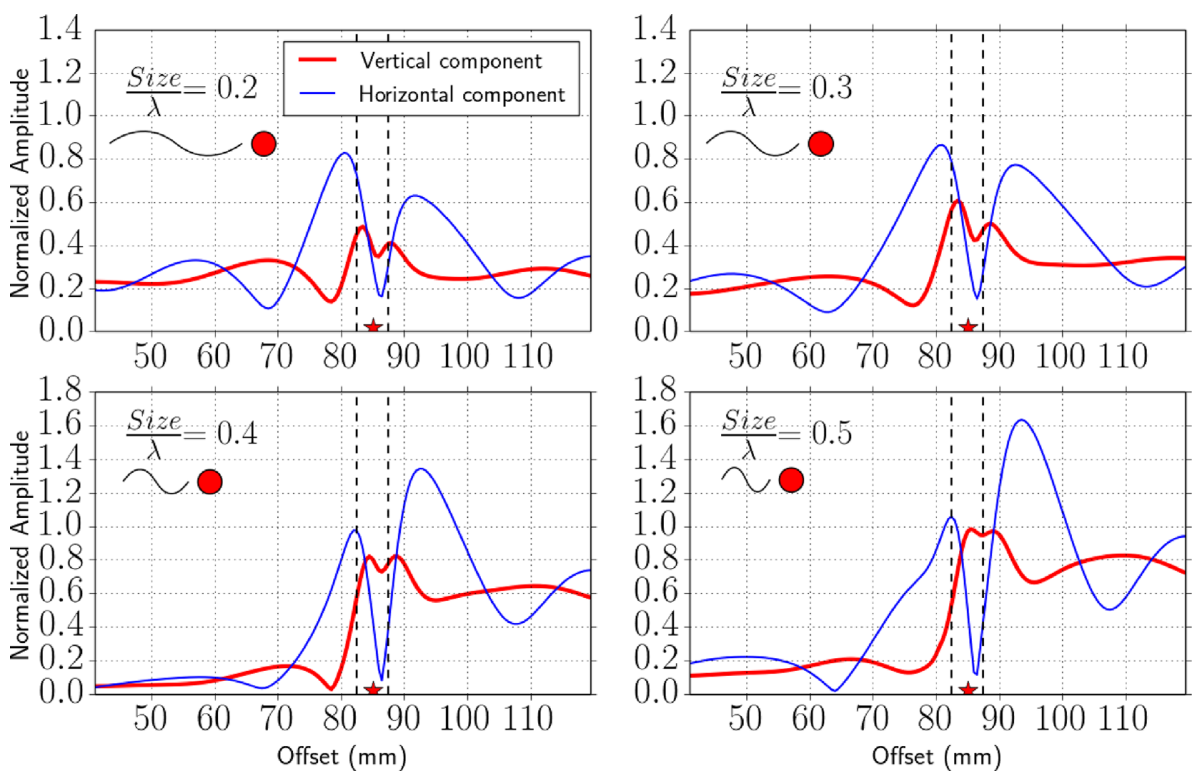

Figure 7. Amplitudes of the diffracted field for a given ratio $\frac{S i z e}{\lambda}$. The cavity's depth is fixed to 0.2 compared to the wavelength. The blue curve corresponds to the H-component and the red one to the V-component. The red star situates the cavity position at the surface.

part of the diffracted wavefield energy would be propagated mainly in the forward direction. Fig. 6(c) presents the corresponding amplitudes for the frequency equal to $100 \mathrm{KHz}$ for the $\mathrm{V}$-component (red curve) and the H-component (blue curve), respectively. They have been extracted from the spectral amplitudes following Fast Fourier Transforms (FFT) of the signals recorded at each receiver position. For each component, all the amplitudes are normalized by the amplitude of the direct wavefield recorded at the first receiver in an equivalent homogeneous medium without cavity. The resulting curves highlight the amplitude variations depending on the offset (source-receivers positions) and can be linked to the shot gather of the parts (a) and (b) of Fig. 6. The main perturbations caused by the cavity (minima and maxima of the curves) are labelled in Fig. 6(c) and recalled on the offset axis in Figs 6(a) and (b). The amplitudes of these extrema on the anomaly curves confirm the stronger effects of the cavity on the H-component compared to the $\mathrm{V}$-component for the diffracted R-wave field. Indeed, at the vicinity of the cavity position (i.e. $5 \mathrm{~m}$ on both sides of it) the amplitude variations are higher for the $\mathrm{H}$-component compared to the vertical one. It can be seen that the amplitude decreases, indicated by A E and A' E', for the offset for which the diffracted modes (body waves and $R$ waves) are split. These modes interfere with each other for particular offsets at the recording surface. The constructive and destructive interferences provide amplitude anomalies, such as seen in the set of points (A A' E E'). They might also be the origin of the amplitude oscillations above and very near the cavity position, as indicated by the points (B B' C C' D D'), where the body waves and $R$ waves are involved together. Fig. 5 shows that the resulting amplitude oscillations due to these interactions dominate for the H-component.

\section{THE EFFECT OF CAVITY SIZE ON THE DIFFRACTED WAVEFIELD}

In order to study the evolution of these features dependent on the cavity diameter in relation to the propagated wavelength noted $\frac{\text { Size }}{\lambda}$, we propose to extract the amplitude curves of the diffracted waves for several $\frac{\text { Size }}{\lambda}$ ratios with a constant cavity roof depth equal to $\frac{\text { Depth }}{\lambda}=0.2$. They were extracted from the spectral amplitudes after fast Fourier transforms (FFT) of the signals recorded at each receiver position. The cavity diameter was modified as the four following configurations: $\frac{\text { Size }}{\lambda}=0.20 ; 0.30 ; 0.40$ and 0.50 . The latter are representative of real cases for addressing subsurface cavities located between 2 and $20 \mathrm{~m}$ depth, with a diameter between 2 and $10 \mathrm{~m}$. For example, a ratio $\frac{\text { Size }}{\lambda}=0.36$ corresponds to a $5 \mathrm{~m}$ wide cavity which is $4 \mathrm{~m}$ deep if the frequency of the propagated seismic $R$ wave is $50 \mathrm{~Hz}$ and for a $R$ waves velocity equals to $700 \mathrm{~m} . \mathrm{s}^{-1}$. The resulting diffraction amplitude curves dependent on the receiver position are presented for these four configurations in Fig. 7. The red curve corresponds to the V-component and the blue one to the $\mathrm{H}$ component of the particular displacement. The centre of the cavity is indicated by the red star and its boundaries by the vertical black dashed lines at the surface. For each case, the maximal amplitudes remain stronger for the $\mathrm{H}$-component than for the vertical one. The level of the amplitude oscillations around the cavity position vary as a function of the $\frac{\text { Size }}{\lambda}$ ratios. The higher the ratio is, the higher the perturbation amplitudes are. Other trends occur as a function of the $\frac{S i z e}{\lambda}$ ratios when the distance to the cavity increases in Fig. 7:

(1) For the lower ratios, that is the cases $\frac{\text { Size }}{\lambda}=0.20$ and $\frac{\text { Size }}{\lambda}=$ 0.30 , the energy is quasi symmetrical on both sides of the cavity.

(2) For the higher ratios, that is the cases $\frac{\text { Size }}{\lambda}=0.40$ and $\frac{\text { Size }}{\lambda}=$ 0.50 , the amplitudes curves are strongly unsymmetrical with higher values behind the void position.

We will call these cases low frequency regime and high frequency regime, respectively. Other numerical tests showed that the limit between these two regimes seems to occur for $\frac{\text { Size }}{\lambda}=0.30$. In order to understand whether these observations recorded at the free surface correspond to a specific phenomenon originating around the cavity, we propose to analyse the behavior of the wavefront for the corresponding frequencies in the cross-section of the space domain. To do this, the total wavefield is computed numerically and the diffracted part is extracted and normalized as explained previously. Then, the V and H-components for $\frac{\text { Size }}{\lambda}=0.2$ and $\frac{\text { Size }}{\lambda}=0.5$ are shown Figs $8(\mathrm{a}, \mathrm{b})$ and $(\mathrm{c}, \mathrm{d})$. The cavity section is drawn as a circular white shape. In order to link each event to the lateral position of the 


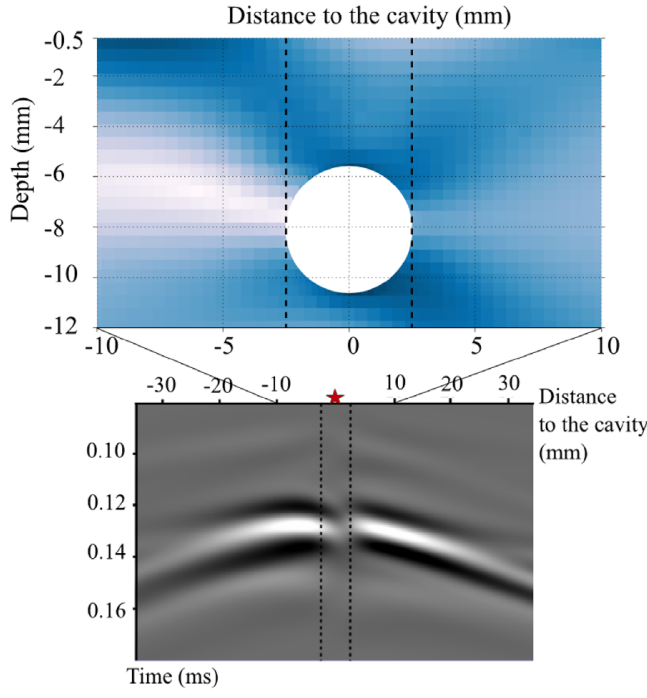

(a)

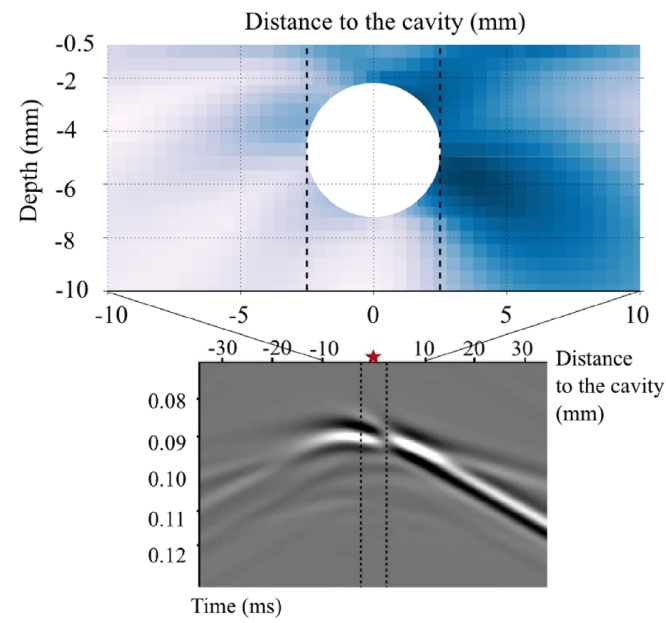

(c)

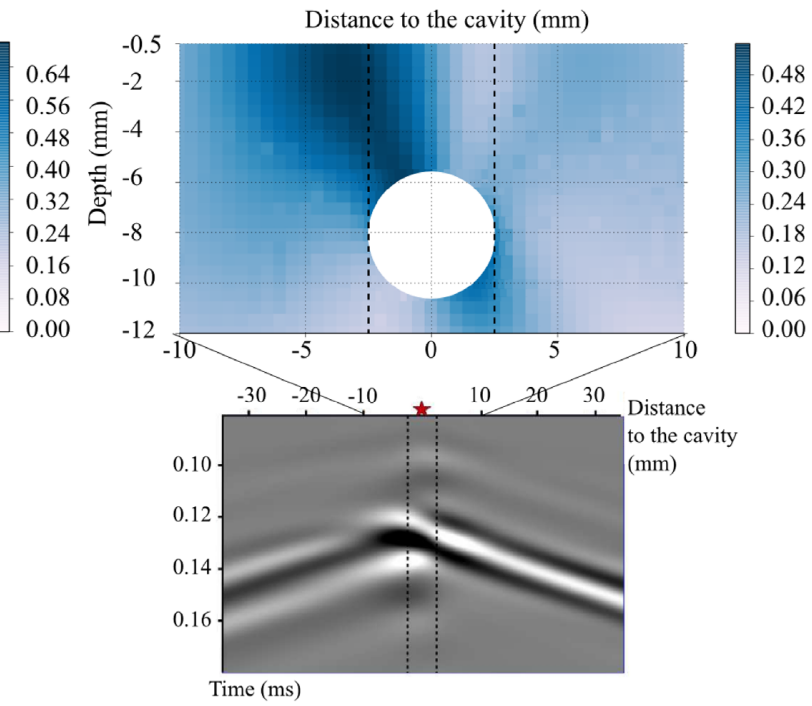

(b)
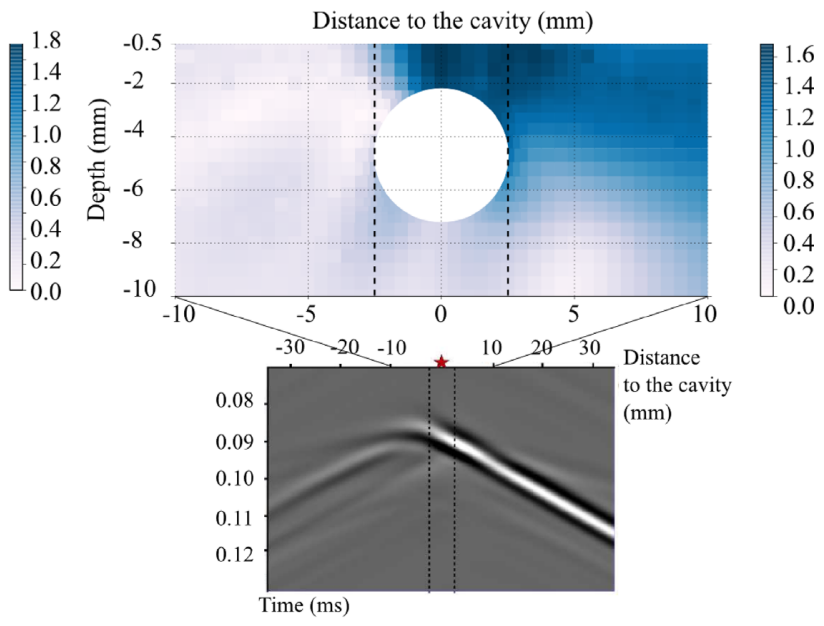

(d)

Figure 8. Normalized diffracted field amplitude in a plane perpendicular to the cavity for low frequency regime (a, b) for the H- and V-components respectively; for a high frequency regime (c, d) for the H- and V-components, respectively; the cavity is represented by the white shape. The recordings of the diffracted wavefield at the surface for a low frequency regime for the $\mathrm{H}$ - and V-components, respectively are represented below the snapshot.

cavity, the wavefield diffracted at the surface and calculated for a Ricker wavelet centred on the corresponding frequency is presented above each snapshot. The frequencies are 50 and $130 \mathrm{kHz}$ for the low and high frequency regimes, respectively. The ratio $\frac{\text { Depth }}{\lambda}=0.2$ is complied within both configurations. In this process, Fig. 8 represents the energy distribution in the diffraction pattern of a cavity illuminated by $R$ waves in the space domain as a function of the $\frac{\text { Size }}{\lambda}$ ratio. The results show that:

(1) For the low frequencies regime (Figs $8 \mathrm{a}$ and b): the diffracted field recorded at the surface appears symmetrical for the Vcomponent. The energy is higher before the void for the $\mathrm{H}$ component.

(2) For the high frequencies regime (Figs $8 c$ and d): the energy of the diffracted field appears to be clearly contained in the forward scattering for the scattering propagated above the cavity toward the free surface. Indeed, the backscattering is clearly attenuated from the offset where the converted $P$ wave appears for each component (i.e. $10 \mathrm{~mm}$ from the near face of the cavity). The local attenuation visible on the surface record for the H-component above the cavity is also visible to $1.5 \mathrm{~mm}$ depth. The converted body-waves are also remarkable on the cross-section.

Thus, much information on the cavity is contained inside the forward scattering. This trend is in agreement with the field data where the backscattering is almost invisible. This may be due to the attenuation of the media, but also, as previously outlined, because a large part the diffracted energy does not return in the direction of the source for a certain size of cavity compared to the wavelength, especially when $0.3>\frac{\text { Size }}{\lambda}>0.5$ as in our case.

\section{THE DIRECT R-FIELD: SOURCE OF INFORMATION ON CAVITY PARAMETERS}

As shown in the previous part, it is crucial to take into account forward scattering for cavity detection because it contains a large part of the diffraction signature. However, the extraction of the diffracted field from the global signal is not easy with field data. Conventional methods using S-transform and $F-K$ filters (e.g. Shao et al. 2016) 


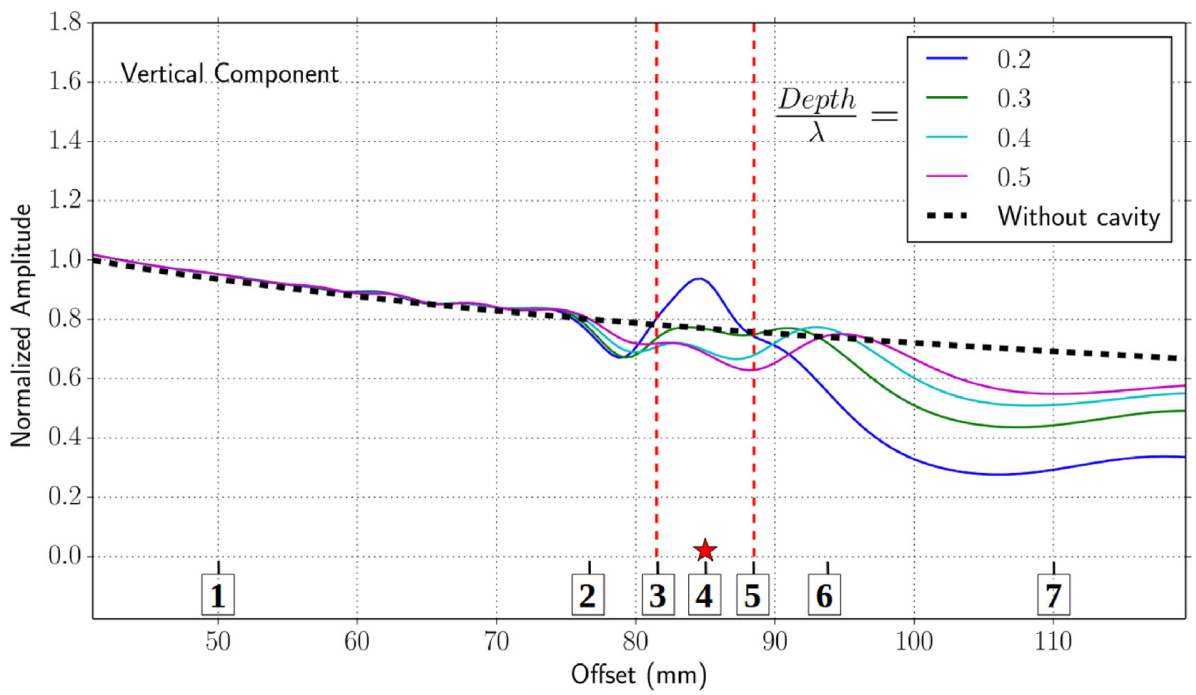

(a)

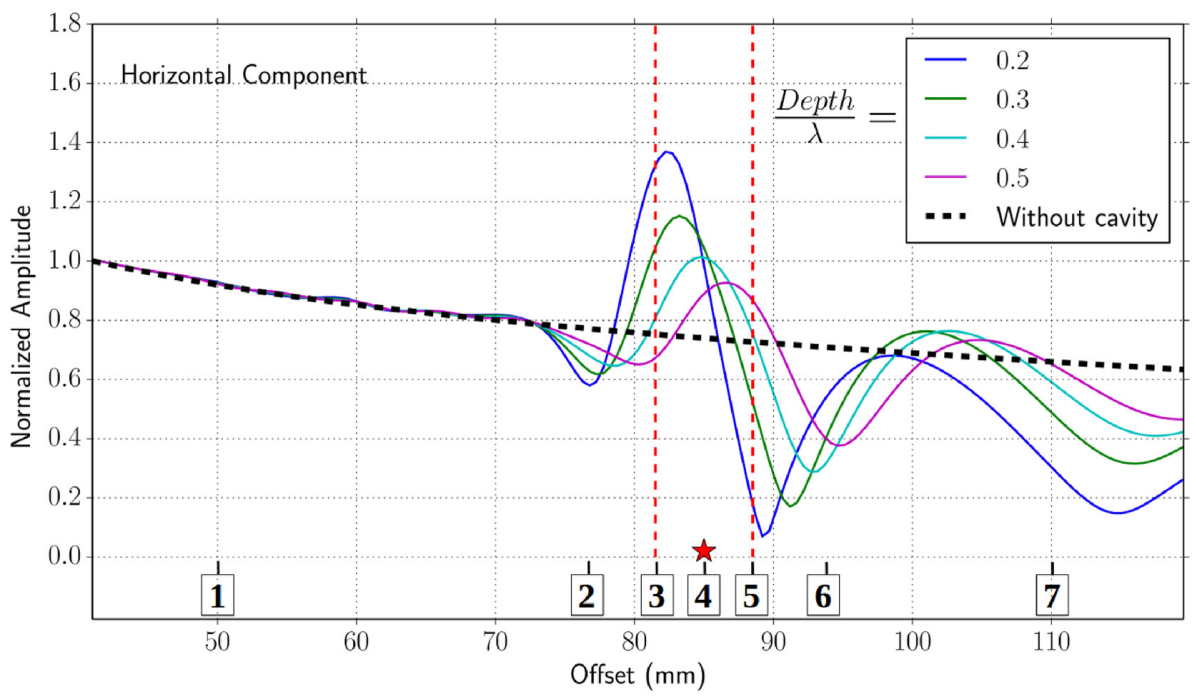

(b)

Figure 9. Amplitude of the direct wave for a given $\frac{\text { Depth }}{\lambda}$ : (a) V-component (b) H-component ; The localization of the cavity centre is represented by the red star. The dashed black line corresponds to the amplitude of the direct wave in a medium without cavity. Specific offsets are marked by different numbers: (1) $50 \mathrm{~mm}$ position distant from the cavity, (2) $76.5 \mathrm{~mm}$ at $5 \mathrm{~mm}$ of the cavity boundary close to the source, (3) $81.5 \mathrm{~mm}$ position of the cavity boundary close to the source, (4) $85 \mathrm{~mm}$ position of the centre of the cavity, (5) $88.5 \mathrm{~mm}$ at $5 \mathrm{~mm}$ position of the cavity boundary far from the source, (6) $93.5 \mathrm{~mm}$ at $5 \mathrm{~mm}$ of the cavity boundary far from the source and (7) $110 \mathrm{~mm}$ position distant from the cavity.

allow isolating the backscattering but they cannot separate the forward scattering from the incident wave.

For these reasons, the study presented in this part is focused on the direct wave which contains the incident wave and the interactions between this field and the forward scattering. More precisely, the aim of this approach is to study if the interactions between the forward scattering and the incident $R$ waves modify the anomalies highlighted in the previous part. That is why we propose to analyse them in the HF context for which the major part of diffracted energy is contained in the forward scattering. More specifically, the cavity size is fixed and equal to $\frac{\text { Size }}{\lambda}=0.5$. Thus, the same homogeneous medium as before is used with the properties of the LAB1000 resin. The measurement setup allows us to window the direct wave by using a mute. The amplitude of the direct $R$ wave at the surface is studied for the two components of displacement as a function of cavity depth in four configurations: $\frac{\text { Depth }}{\lambda}=0.2 ; 0.3 ; 0.4 ; 0.5$ for analysing the sensitivity of the cavity signature on the direct wave as a function of the depth of the void. The results are presented in Fig. 9. The amplitudes are normalized by the amplitude of the first trace in an equivalent medium without cavity for a given frequency. The black dashed line is the reference amplitude curve of the direct $R$ wave in a medium without cavity. The projection of the cavity boundaries are represented by the red dashed lines.

For the low offsets, the reference curve obtained in a medium without cavity and the other curves obtained in a medium with cavity at different depths are superimposed. The cavity signature on the direct wave is only visible at about $10 \mathrm{~mm}$ (almost one wavelength) before the location of the centre of the cavity for each component and for all depths. It is important to note the strong variations of amplitudes before and after the void. These variations are marked by an increase of amplitudes, followed by oscillations for positions above the cavity. All the perturbations are more pronounced on the 

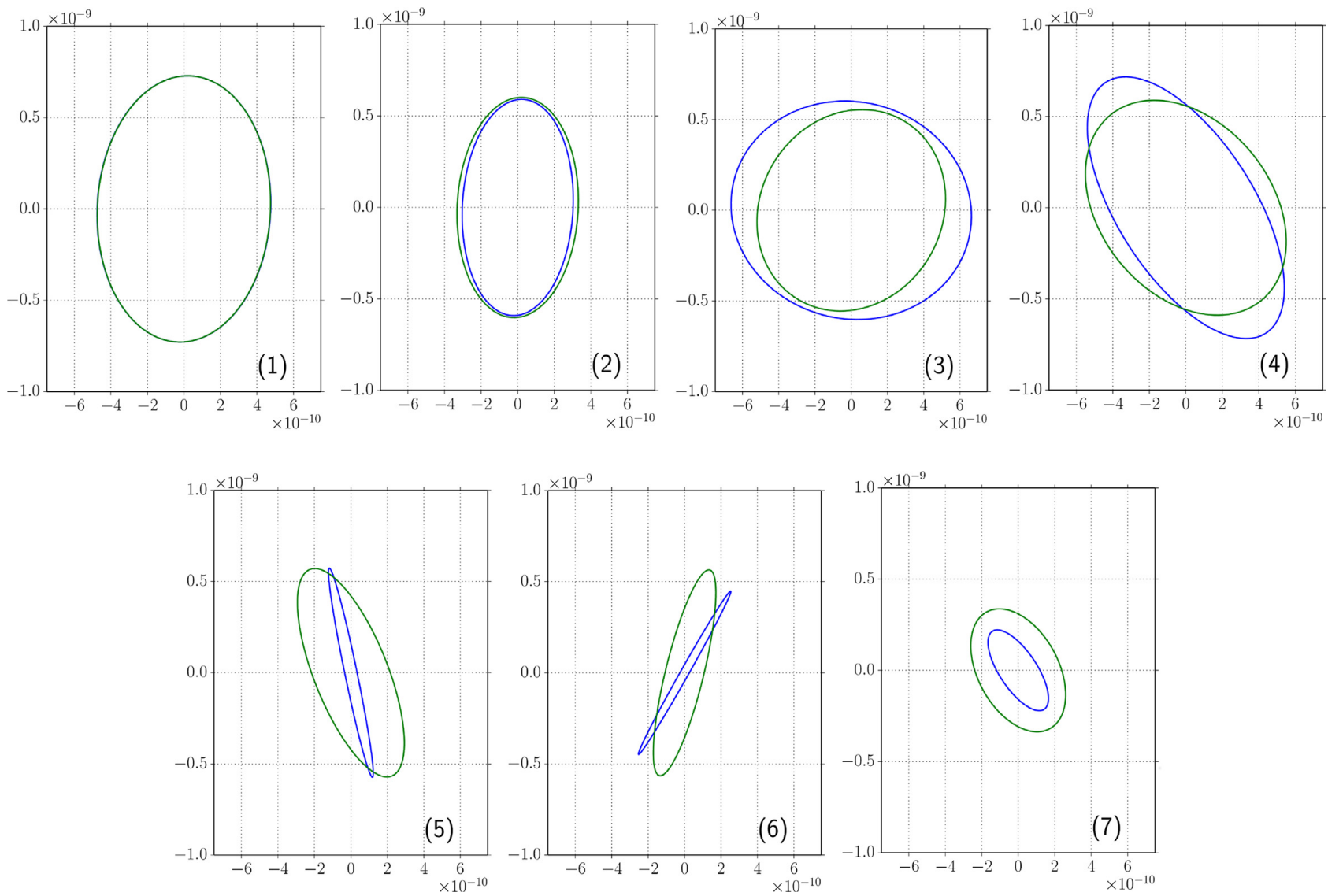

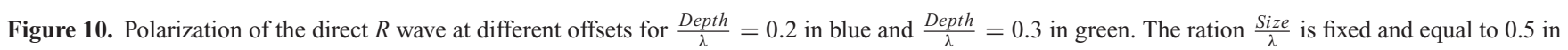
each case.

Table 2. Overview of the results.

Seismic features

Scattering features

Effect on cavity size on the $R$ wave scattering part

Effect on cavity depth on the direct $R$ wave
Observations

- Sensibility of the H-component to the cavity

- Asymmetric diffraction shape

- Diffraction hyperbola curved on the H-component

- Energy quasi symmetrical on both sides of the cavity for small cavities sizes

- Forward scattering amplitudes stronger than back scattering amplitudes for higher cavities sizes

- Anomalies H/V which decrease with the cavity depth

- Effect on the elliptic particule displacement
Phenomena interpretation

- Mode conversions more visible on the H-component

- Influence of body waves in the near-field of the cavity

- Low frequency regime: $0.20<\frac{\text { Size }}{\lambda}<0.30$

- High frequency regime: $0.40<\frac{\text { Size }}{\lambda}<0.50$

- Interactions between scattered and direct field

- Mix of $R$ waves and body waves which creates circular motion or only vertical motion
H-component and all these disturbances in the vicinity of the cavity are due to the interactions between the direct and the diffracted fields. These interferences, constructive or destructive, depend on the phase difference between the different fields included in the conversion modes. For the cases where $\frac{\text { Depth }}{\lambda}=0.2$ and $\frac{\text { Size }}{\lambda}=0.5$ as previously shown in Fig. 7, the energy of the diffracted field is focused behind the void with high amplitude. However, the amplitudes of the direct $R$ wave for this configuration in Fig. 9 are low and out of phase compared to Fig. 7. This is due to the fact that the interactions between the forward scattering and the incident wave are mostly destructive. Moreover, the positions of the maximal and minimal energies on the $\mathrm{H}$-component for the direct $R$ wave depend on the ratio $\frac{\text { Depth }}{\lambda}$. They are shifted towards the high offsets proportionally to $\frac{\text { Depth }}{\lambda}$ for the ratio range $[0.2 ; 0.5]$ Moreover, the direct
$R$ wave undergoes energy loss behind the cavity ( $88 \mathrm{~mm}$ to $95 \mathrm{~mm}$ ) compared to the amplitude of the direct $R$ wave in a medium without cavity. This is clearly identifiable on the two components. The deeper the void is, the lower the energy loss. This can be caused by the decrease of the incident wave energy with depth which depends on the eigenfunction of the $R$ waves for the $\mathrm{V}$ - and $\mathrm{H}$-displacement.

The study of the direct $R$ wave amplitudes showed particularities as a function of the V-and $\mathrm{H}$-components. The anomalies of these components caused by the cavity can be studied together based on the variations of the elliptical particle motion recorded at the surface. Fig. 10 presents the ellipses calculated for $\frac{\text { Depth }}{\lambda}=0.2$ and 0.3 , respectively, in blue and green. They are calculated with the amplitude of the incident R-field without normalization. Numbers 1 to 7 indicated in Fig. 10 correspond to the specific offsets also 


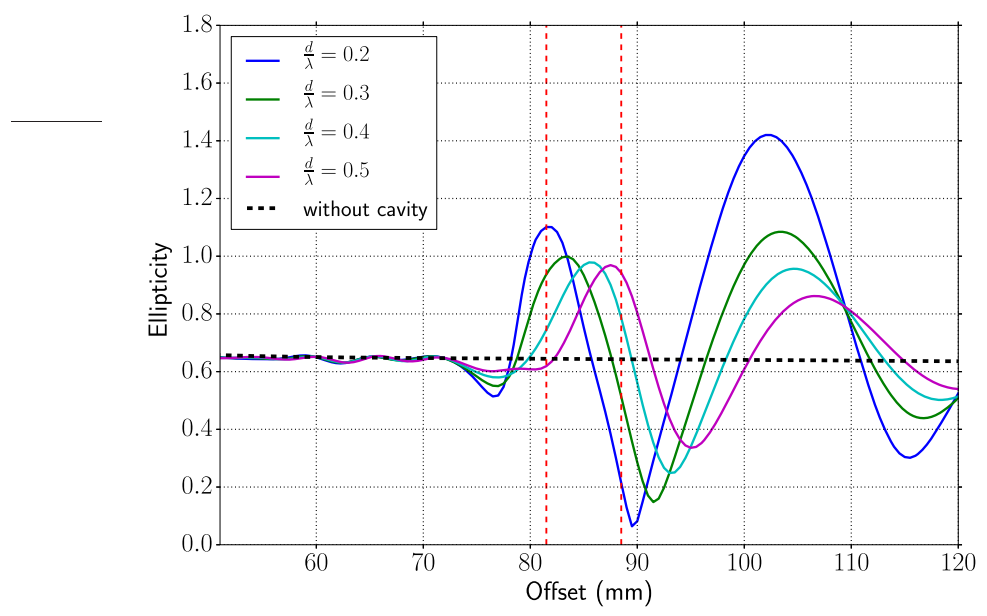

Figure 11. $\mathrm{H} / \mathrm{V}$ ratios of the direct $R$ wave at different offsets for different $\frac{\text { Depth }}{\lambda}$. Size equal to 0.5 . The dashed black line corresponds to the H/V ratio of the direct $R$ wave in a medium without cavity. The cavity's boundaries at the surface are delimited by the vertical red dashed line.

indicated in Fig. 9. For the lowest offsets, numbers 1 and 2, the cavity has no impact on the direct wave and the particule displacement is elliptical. The perturbation begins to be identifiable next to the cavity boundary closest to the source (number 3 ). For this offset, the amplitude on the $\mathrm{H}$-component increases and the resulting particule displacement is almost circular. At this specific location, part of the diffracted wavefield is recorded with a hyperbolic parametrization of the arrival time for the H-component in Fig. 6 (a). Consequently, the body waves mostly contribute to the circular polarization. However, when the polarization is calculated next to the cavity boundary farthest from the source, the amplitude on the H-component decreases, the minor axis of the ellipse is smaller and its shape is flatter. Note that for this offset position, the energy of the diffracted wavefield is maximal (Fig. 7). That indicates that the effect of the cavity on the direct wave is due to the destructive interferences between the direct and the diffracted wavefields. The direct $R$ wave bears the cavity signature for the offsets far from the heterogeneity. Its amplitudes can be directly linked to the cavity depth provided that a sufficient proportion of the energy contained in the $R$ waves encounters the cavity.

\section{DISCUSSION AND PERSPECTIVES}

The reduced-scale measurements carried out at the surface of the resin material allowed us to validate the reliability of modeling a medium containing a void by numerical simulations. To perform the comparison, the spectral ratio method was used to determine the intrinsic attenuation in the resins and propose a strong hypothesis of constant quality factors. This assumption could be questioned in the frequency band used, particularly for the shallower layer. Although the numerical simulations showed good agreement with the reduced-scale experiments (Fig. 2), a slight shift in time and amplitude could persist between the two sets of data. However, this combined approach allowed assessing the robust seismic observables available in $\mathrm{V}$ - and $\mathrm{H}$-components usable for cavity detection. First, the reduced-scale experiments that we carried out on a heterogeneous and dispersive medium defined observables that were robust enough for use in the field. Then, the numerical simulations on a homogeneous medium quantified these observables solely as a function of the cavity's depth and size. The different results recapitulated in the Table 2 highlighted the need to take the H-component into consideration during field acquisitions as it is more sensitive to cavities than the V-component. Indeed, the recordings of the H-component showed converted body waves with have strong amplitudes in comparison to the waves amplitudes recorded on the V-component. This conferred a hyperbolic shape to the diffracted wave field that was not present on the V-component, due to the linear displacement of the Rayleigh wave at the surface (Fig. 6).

These observations led us to describe the phenomena as follows: when the $R$ waves reach the cavity, the diffracted wavefield is split in two diffracted parts, that is R-diffracted waves and body-diffracted waves. As observed previously, the hyperbola shape of the diffraction pattern indicated that most of the energy is propagated into body waves. When the latter reach the soil surface again, they are reconverted into $R$ waves. Thus, for larger offsets, the diffracted waves from $R$ waves recorded at the surface involve three kinds of waves linked to the diffraction and conversion phenomena:

(1) R-R mode: incident $R$ waves diffracted in $R$ waves.

(2) R-B mode: incident $R$ waves diffracted in converted body waves.

(3) R-B-R mode: incident $R$ waves first diffracted in converted body-waves and then converted into $R$ waves at the free surface (for higher offsets).

We showed that the field energy of the diffracted wave exhibited significant disturbances due to interactions with these converted body waves. This led us to define two main states as a function of the cavity diameter over the wavelength ratio. The high frequency state gathers the diffracted energy on the front, whereas the low frequency state displays a homogeneous energy distribution for the V-component around the void for high offsets. To sum up, the larger the cavity compared to the wavelength, the more the energy is propagated in the forward direction. At first sight this result seems to be in contradiction with the observations carried out by Chai et al. (2012). Their studies, conducted on the relationship between cavity size and wavelength led to the conclusion that back-scattering energy increases with the $\frac{\text { Size }}{\lambda}$ ratios. To come to these results, they made their observations at $5 \mathrm{~m}$ from the near face of the cavity. The same observations can be made in Fig. 7. At the vicinity of the cavity (i.e. $5 \mathrm{~m}$ from the cavity's boundaries) the perturbations of the diffracted field increase with the diameter of the void on each component (events B D and B' D' on the Fig. 6). However, for a higher offset, the general trend clearly shows that the forward scattering has higher amplitudes than the backscattering when the 


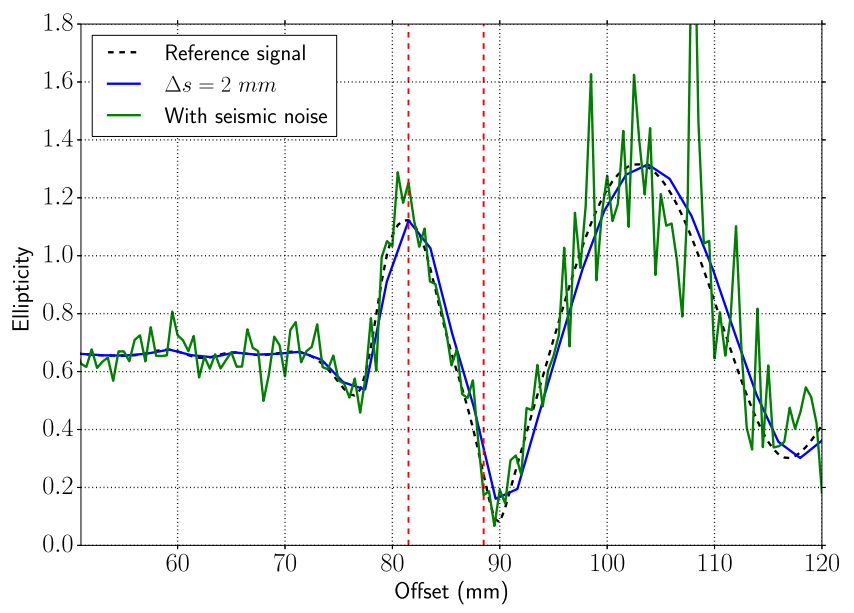

Figure 12. $\mathrm{H} / \mathrm{V}$ ratios of direct $R$ wave versus offsets for $\frac{\text { Depth }}{\lambda}=0.2$ and $\frac{\text { Size }}{\lambda}=0.3$. The dashed black line corresponds to the reference signal in a homogeneous space with a circular cavity. The green line was obtained by adding 40 and 20 per cent of Gaussian noise on H- and V-component, respectively, with a noise bandlimited from 10 to $160 \mathrm{~Hz}$. The blue line was obtained with a trace increment equal to $\Delta s=2 \mathrm{~mm}$. The cavity's boundaries at the surface are delimited by the vertical red dashed line.

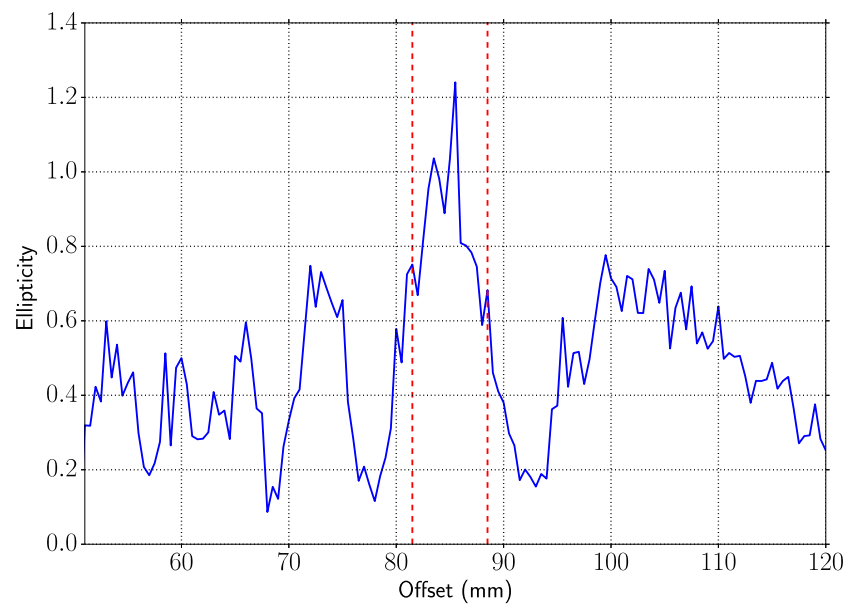

Figure 13. H/V ratios of the total wavefield at different offsets obtained in the experimental Bicperc medium. $\frac{\text { Size }}{\lambda}=0.36$ and $\frac{\text { Depth }}{\lambda}=0.42$. The cavity's boundaries at the surface are delimited by the vertical red dashed line.

$\frac{\text { Size }}{\lambda}$ ratios increase. At the vicinity of the cavity, this study confirmed the results already demonstrated by the authors mentioned above. In addition, by taking into account a wide range of offsets and thus mode conversions, we highlighted two different behaviours as a function of cavity size: the low frequency regime and the high frequency regime.

These results may explain the low backscattering energy in some field data in the presence of a cavity. As most of the information is contained in the diffracted field behind the cavity, we studied the direct Rayleigh wave, which combines the incident field and forward scattering. High amplitude anomalies on this direct field are caused by the cavity. These anomalies are found above the void and about one wavelength behind it. Their presences are directly related to the cavitys depth and also impact $R$ wave polarization (Fig. 10). However, it can be difficult to work with absolute amplitude with field data since the coupling between the soil and the receivers is unknown, as is the intrinsic attenuation of the media. To avoid all these issues, we studied the amplitudes for the two components relative to each other. The results are presented in the form of $\mathrm{H} / \mathrm{V}$ ratio (ellipticity).

Commonly, the $\mathrm{H} / \mathrm{V}$ ratio technique is applied to investigate the spectral ratio between the $\mathrm{H}$ - and the $\mathrm{V}$-component of ambient vibrations (Nakamura 1989; Lachet et al. 1994; Fäh et al. 2001; Molnar et al. 2018). It is used to determine the fundamental site resonance frequency, which can be used to estimate the sediment thickness and depth to bedrock in a tabular medium (Lane et al. 2008; Lontsi et al. 2015). Recently, Kolesnikov \& Fedin (2018) have applied the $\mathrm{H} / \mathrm{V}$ method for the underground cavities detection by using body waves. The distribution of frequencies and amplitudes of extracted standing waves in the space between the ground surface and the upper face of the cavity enables to determine cavity's boundaries. However, this method supposes to have a priori information on the object position, to know the velocity of the medium and to record body waves at the near surface which are, in most of the case, not predominant. Here, we propose an other way to use the H/V ratio. The ellipticity is calculated by using a controlled-source for one frequency and correspond to a unique $\frac{\text { Size }}{\lambda}$ and $\frac{\text { Depth }}{\lambda}$ (Fig. 11). The $\mathrm{H} / \mathrm{V}$ ratios are calculated for the incident $\mathrm{R}$-wave by varying the cavity depth depending on the wavelength and by fixing the ratio $\frac{\text { Size }}{\lambda}=0.5$ as done previously. The global maximum of the $\mathrm{H} / \mathrm{V}$ ratio is located just behind the cavity's boundary near the source and for offsets from 97 to $108 \mathrm{~mm}$. This is revealed by strong anomalies of the $\mathrm{H} / \mathrm{V}$ ratio that can be two times higher than $\mathrm{H} / \mathrm{V}$ values without a cavity. Just above the void, the $\mathrm{H} / \mathrm{V}$ values tend towards 1 which indicates a circular ellipse visible on Fig. 10 number 3. The anomalies are all caused by the interactions between the direct- $R$ wave and the converted body waves. The offsets where the perturbations of the $\mathrm{H} / \mathrm{V}$ ratio occur depend on the cavity depth and appear to be good indicators for cavity detection. Although these anomalies are smaller for higher $\frac{D e p t h}{\lambda}$ ratios, they are remarkable at different offsets and the effects of the cavity are visible at almost one wavelength behind the object. By tracing the ellipticity curves computed along a profile we demonstrate that they are good indicators of the existence of an underground cavity. Furthermore, this method overcomes certain difficulties encountered in the field due to difficult terrains. Fig. 12 represents the $\mathrm{H} / \mathrm{V}$ ratios of direct $R$ wave versus offsets for $\frac{\text { Depth }}{\lambda}=0.2$ and $\frac{\text { Size }}{\lambda}=0.3$. The dashed black line corresponds to the reference signal in a homogeneous space with 
a circular cavity. The green line was obtained by adding 40 and 20 per cent of gaussian noise on $\mathrm{H}$ - and V-component, respectively, with a noise band limited from 10 to $160 \mathrm{~Hz}$. The blue line was obtained with a trace increment equal to $\Delta s=2 \mathrm{~mm}$ instead of the $0.5 \mathrm{~mm}$ used for the reference case. The three curves have the same global shape: we can see that the anomalies at the vicinity of the cavity seem to be robust to the presence of noise in the signal and to a spaced receivers network.

The $\mathrm{H} / \mathrm{V}$ ratio versus the offsets was also calculated in the experimental model Bicperc Fig. 13 without source estimation in order to approach real field conditions. The ratios $\frac{\text { depth }}{\lambda}$ and $\frac{\text { size }}{\lambda}$ are, respectively, equal to 0.42 and 0.36 by taking into account a frequency equal to $100 \mathrm{kHz}$ and the velocity of the second layer in this medium. With these experimental data, it remains difficult to extract the backscattering from the direct $R$ waves. Thus, the ellipticity is calculated for the total R-field to approximate real field conditions again. The boundaries of the cavity are indicated by the red dashed line. For the lowest offsets, the variations of the ellipticity correspond to the recording of the backscattering. Just above the void, a peak of ellipticity is clearly visible with values up to 1 which means that the H-component amplitude is higher than the V-component one. This peak is followed by a decrease and a second peak behind the void that we can also clearly distinguish with numerical data Fig. 11. This analysis provides informations about the lateral position of the void. This first step of analysis on numerical data including random noise as well as on experimental data contribute to confirm the robustness of the H/V observable to provide informations for detecting an underground cavity and describing some of its geometrical parameters. This is encouraging for further development towards a new methodology of imaging. In this way, further works will be conducted to test the sensitivity of this observable in more complex media, for example by considering the rugosity of the cavity boundaries as well as in context of complex field data including an heterogeneous surrounding medium. Moreover, the 3-D effects of a closed cavity would be interesting to analyse. It should involve other conversion modes, for example to Love waves, thus it needs a complete analysis, which will be considered in further works.

\section{ACKNOWLEDGEMENTS}

We would like to thank the creators of the EFISPEC3D Spectral Element Method modelling code. We are also grateful to the CCIPL (Centre de Calcul Intensif des Pays de la Loire) for providing access to its high-performance computing facilities and the support given by its staff. This work was cofounded by IFSTTAR, BRGM and Région Pays de la Loire.

\section{REFERENCES}

Almuhaidib, A.M. \& Toksz, M.N., 2015. Imaging of near-surface heterogeneities by scattered elastic waves, Geophysics, 80(4), 83-88.

Bishop, I., Styles, P., Emsley, S.J. \& Ferguson, N.S., 1997. The detection of cavities using the microgravity technique: Case histories from mining and karstic environments, Geol. Soc. Lond. Eng. Geol. Spec. Publ., 12(1), 153-166.

Bitri, A., Samyn, K. \& Filippi, C., 2016. Can we really detect cavities using seismic Rayleigh waves?, Near Surface Geoscience 2016-22nd European Meeting of Environmental and Engineering Geophysics, EAGE.

Belfer, I., Bruner, I., Keydar, A., Kravtsov, A. \& Landa, E., 1998. Detection of shallow objects using refracted and diffracted seismic waves, J. appl. Geophys., 38(3), 155-166.
Berkovitch, A., Belfer, I., Hassin, Y. \& Landa, E., 2009. Diffraction imaging by multifocusing, Geophysics, 74(6), 75-81.

Blonk, B. \& Herman, G.C., 1994. Inverse scattering of surface waves: a new look at surface consistency, Geophysics, 59(6), 963-972.

Bretaudeau, F., Brossier, R., Leparoux, D., Abraham, O. \& Virieux, J., 2013. 2D elastic full-waveform imaging of the near-surface: application to synthetic and physical modelling data sets, Near Surf. Geophys., 11(3), 307316.

Bretaudeau, F., Leparoux, D., Durand, O. \& Abraham, O., 2011. Small-scale modeling of onshore seismic experiment: a tool to validate numerical modeling and seismic imaging methods, Geophysics, 76(5), 101-112.

Butler, D.K., 1984. Microgravimetric and gravity gradient techniques for detection of subsurface cavities, Geophysics, 49, 1084-1096.

Campman, X.H., van Wijk, K., Scales, J.A. \& Herman, G.C., 2005. Imaging and suppressing near-receiver scattered surface waves, Geophysics, 70(2), 21-29.

Cardarelli, E., Marrone, C. \& Orlando, L., 2002. Evaluation of tunnel stability using integrated geophysical methods, J. appl. Geophys., 52(2-3), 93-102.

Chai, H., Phoon, K., Goh, S. \& Wei, C., 2012. Some theoretical and numerical observations on scattering of Rayleigh waves in media containing shallow rectangular cavities, J. appl. Geophys., 83, 107-119.

De Martin, F., 2010. Influence of the nonlinear behavior of soft soils on strong ground motion, Doctoral dissertation, Ecole Centrale Paris, France.

Dravinski, M. \& Yu, M.C., 2013. The effect of impedance contrast upon surface motion due to scattering of plane harmonic P, SV and Rayleigh waves by a randomly corrugated elastic inclusion, J. Seismol., 17(2), 281-295.

Ernst, F.E., Herman, G.C. \& Ditzel, A., 2002. Removal of scattered guided waves from seismic data, Geophysics, 67(4), 1240-1248.

Fäh, D., Kind, F. \& Giardini, D., 2001. A theoretical investigation of average $\mathrm{H} / \mathrm{V}$ ratios, J. geophys. Int., 145, 535-549.

Fehdi, C., Nouioua, D., Djabri, L. \& Salameh, E., 2014. Detection of underground cavities by combining electrical resistivity imaging and ground penetrating radar surveys: a case study from Draa Douamis a (North East of Algeria), in H2Karst Research in Limestone Hydrogeology, pp. 69-82. Springer, Cham.

Di Fiore, V., Angelino, A., Passaro, S. \& Bonanno, A., 2013. High resolution seismic reflection methods to detect near surface tuff-cavities: a case study in the Neapolitan area, Italy, J. Cave Karst Stud., 75(1), 51-59.

Foti, S., Parolai, S., Albarello, D. \& Picozzi, M., 2011. Application of surface wave methods for seismic site characterization, Surv. Geophys., 32(6), 777-825.

Foti, S., Comina, C., Boiero, D. \& Socco, L.V., 2009. Non-uniqueness in surface-wave inversion and consequences on seismic site response analyses, Soil Dyn. Earthq. Eng., 29, 982-993.

Gelis, C., Leparoux, D., Virieux, J., Bitri, A., Operto, S. \& Grandjean, G., 2005. Numerical modeling of surface waves over shallow cavities, $J$. Environ. Eng. Geophys., 10(2), 111-121.

Gelis, C., 2005. Inversion des formes d'onde lastique dans le domaine espace-frquence en deux dimensions. Application la caractrisation de la subsurface dans le cadre de la dtection de cavit souterraine, Doctoral dissertation, Universit de Nice-Sophia Antipolis, France.

Grandjean, G. \& Leparoux, D., 2004. The potential of seismic methods for detecting cavities and buried objects: experimentation at a test site, $J$. appl. Geophys., 56(2), 93-106.

Gucunski, N., Ganji, V. \& Maher, M.H., 1996. Effects of obstacles on Rayleigh wave dispersion obtained from SASW test, Soil Dyn. Earthq. Eng., 15(4), 223-231.

Herman, G.C., Milligan, P.A., Huggins, R.J. \& Rector, J.W., 2000. Imaging shallow objects and heterogeneities with scattered guided waves, Geophysics, 65(1), 247-252.

Hyslop, C. \& Stewart, R.R., 2015. Imaging lateral heterogeneity using reflected surface waves, Geophysics, 80(3), 69-82.

Kaslilar, A., 2007. Inverse scattering of surface waves: imaging of nearsurface heterogeneities, J. geophys. Int., 171(1), 352-367. 
Kolesnikov, Y.U. \& Fedin, K.V., 2018. Detecting underground cavities using microtremor data: physical modelling and field experiment, Geophys. Prospect., 66(2), 342-353.

Komatitsch, D. \& Tromp, J., 1999. Introduction to the spectral element method for three-dimensional seismic wave propagation, J. geophys. Int., 139(3), 806-822.

Korneev, V., 2009. Resonant seismic emission of subsurface objects, Geophysics, 74(2), 47-53.

C Lachet, C. \& Bard, P.Y., 1994. Numerical and theoretical investigations on the possibilities and limitations of Nakamura's technique, J. Phys. Earth, 42(5), 377-397.

Lane, J.W.Jr., White, E.A., Steele, G.V. \& Cannia, J.C., 2008. Estimation of bedrock depth using the horizontal-to-vertical (H over V) ambient-noise seismic method, in Proceedings of the Symposium on the Application of Geophysics to Engineering and Environmental Problems 2008, pp. 490-502, Society of Exploration Geophysicists.

Leparoux, D., Bitri, A. \& Grandjean, G., 2000. Underground cavity detection: a new method based on seismic Rayleigh waves, Eur. J. Environ. Eng. Geophys., 56(2), 93-106.

Liu, Z., AlTheyab, A., Hanafy, S.M. \& Schuster, G., 2017. Imaging nearsurface heterogeneities by natural migration of backscattered surface waves: field data test, Geophysics, 82(3), 197-205.

Lontsi, A.M., Sànchez-Sesma, F.J., Molina-Villegas, J.C., Ohrnberger, M. \& Krüger, F, 2015. Full microtremor $\mathrm{H} / \mathrm{V}(\mathrm{z}, \mathrm{f})$ inversion for shallow subsurface characterization, J. geophys. Int., 202(1), 298-312.

Luke, B.A. \& Chase, D.S., 1997. Detecting caves using seismic surface waves: a feasibility study, in The Engineering Geology and Hydrogeology of Karst Terranes, pp. 419-424, eds ,Beck, F.B. \& ,Stephenson, J.B., Rotterdam.

Maraio, S. \& Bruno, P.P.G., 2015. Near-surface voids in the Neapolitan Volcanic Tuff (Italy) detected by seismic refraction tomography, in Proceedings of the 21nd European Meeting of Environmental and Engineering Geophysics: Near Surface Geoscience 2015, Turin, Italie.

Miller, R.D. \& Steeples, D.W., 1991. Detecting voids in a $0.6 \mathrm{~m}$ coal seem, $7 \mathrm{~m}$ deep, using seismic reflection, in SEG Technical Program Expanded Abstracts, pp. 109-119, Society of Exploration Geophysicists.

Mochales, T., Casas, A.M., Pueyo, E.L., Pueyo, O., Romàn, M.T., Pocovì, A., Soriano, M.A. \& Ansòn, D., 2007. Detection of underground cavities by combining gravity magnetic and ground penetrating radar surveys: a case study from the Zaragoza area, NE Spain, Environ. Geol., 53(5), 1067-1077.

Molnar, S. et al., 2018. Application of microtremor horizontal-to-vertical spectral ratio (MHVSR) analysis for site characterization: State of the art, Surv. Geophys., 39(4), 613-631.

Nakamura, Y., 1989. A method for dynamic characteristics estimations of subsurface using microtremors on the ground surface, Quart. Rep. RTRI Jpn., 30, 25-33.

Nasseri-Moghaddam, A.,Cascante,G. Phillips C. \& Hutchinson, D.J., 2007. Effects of underground cavities on rayleigh waves-field and numerical experiments, Soil Dyn. Earthq. Eng., 27(4), 300-313.

Pageot, D., Leparoux, D., Le Feuvre, M., Durand, O., Cte, Ph. \& Capdeville, Y., 2017. Improving the seismic small-scale modelling by comparison with numerical methods, J. geophys. Int., 211(1), 637-649.

Park, C.B., Miller, R.D. \& Xia, J., 2009. Detection of near-surface voids using surface waves, in 22nd Proceeding of the Symposium on the Application of Geophysics to Engineering and Environmental Problems, pp. 291-286, Society of Exploration Geophysicists.

Perez Solano, C., 2012. Imagerie sismique de la proche sub-surface : modification de l'inversion des formes d'onde pour l'analyse des ondes de surface, Doctoral dissertation, Mines Paris tech, France.

Peters, L.Jr., Daniels, J.J. \& Young, J.D., 1999. Seismic waveform inversion in the frequency domain, Part 1: theory and verification in a physical scale model, Geophysics, 64(3), 888-901.

Putnam, N.H., Nasseri-Moghaddam, A., Kovin, O., Torgashov, E. \& Anderson, N.L., 2008. Analysis using surface wave methods to detect shallow manmade tunnels, Technical Report, Department of Geological Sciences Missouri University of Science and Technology, Rolla, Missouri.

Riddle, G.I., Hickey, C.J. \& Schmitt, D.R., 2010. Subsurface tunnel detection using electrical resistivity tomography and seismic refraction tomography: a case study, in Proceedings of the 23rd Symposium on the Application of Geophysics to Engineering and Environmental Problems, pp. 552-562.

Romdhane, A., Grandjean, G., Brossier, R., Réjiba, F. \& Operto, S. 2011. Shallow-structure characterization by $2 \mathrm{D}$ elastic full-waveform inversion, Soc. Explor. Geophys., 76(3), 81-93.

Satoh, T., Yamagata, K., Poran, C.J. \& Rodriguez, J.A., 1991. Soil profiling by spectral analysis of surface waves, in International Conferences on Recent Advances in Geotechnical Earthquake Engineering and Soil Dynamics.

Schneider, F.M., Esterhazy, S., Perugia, I. \& Bokelmann, G., 2017. Seismic resonances of spherical acoustic cavities, Geophys. Prospect., 65, 1-24.

Shao, G., Tsoflias, G.P. \& Li, C., 2016. Detection of near-surface cavities by generalized S-transform of Rayleigh waves, J. appl. Geophys., 129, 53-65.

Shtivelman, V., 2003. Using surface waves for studying the shallow subsurface, Boolettino di geofisica teorica ed applicata, 44(3-4), 223-236.

Sloan, S.D., Peterie, L.P., Miller, R.D., Ivanov, J., McKenna, J.R., Broadfoot, S.W. \& Metheny, O.M., 2015. Detecting clandestine tunnels using nearsurface seismic techniques, Geophysics, 80(5), 127-135.

Snieder, S., 1987. Surface Wave scattering theory, Doctoral dissertation, University of Utrecht, Pays-Bas.

Socco, L.V. \& Comina, C., 2004. Approximate direct estimate of S-wave velocity model from surface wave dispersion curves, in 21nd European Meeting of Environmental and Engineering Geophysics : Near Surface Geoscience 2015, Turin.

Socco, L.V. \& Strobbia, C., 2004. Surface-wave method for near-surface characterization: a tutorial, Near Surf. Geophys., 2(4), 165-185.

Valensi, R., Leparoux, D., Durand, O., Bretaudeau, F. \& Cte, Ph., 2015. Multicomponent reduced scale seismic modelling: upgrade of the MUSC laboratory with application to polarization observations, J. geophys. Int., 202(3), 1993-2004.

Watkins, J.S., Godson, R.H. \& Watson, K., 1967. Seismic detection of near-surface cavities, U.S.Geol. Surv., Prof. Paper, Astrogeology, doi:10.3133/pp599A.

Xia, J., Nyquist, J.E., Xu, Y. \& Roth, M., 2007. Feasibility of detecting nearsurface feature with Rayleigh-wave diffraction, J. appl. Geophys., 62(3), $244-253$.

Xu, C. \& Butt, S.D., 2006. Evaluation of MASW techniques to image steeply dipping cavities in laterally inhomogeneous terrain, J. appl. Geophys., 59(2), 106-116. 